\title{
BMJ Open Cost-effectiveness of interventions to control cardiovascular diseases and diabetes mellitus in South Asia: a systematic review
}

\author{
Kavita Singh, ${ }^{1,2,3}$ Ambalam M Chandrasekaran, ${ }^{2}$ Soumyadeep Bhaumik, ${ }^{4}$ \\ Kaushik Chattopadhyay, ${ }^{5,6}$ Anuji Upekshika Gamage, ${ }^{7}$ Padmal De Silva, ${ }^{8}$ \\ Ambuj Roy, ${ }^{9}$ Dorairaj Prabhakaran, ${ }^{2,3,6}$ Nikhil Tandon ${ }^{1}$
}

To cite: Singh $\mathrm{K}$,

Chandrasekaran AM, Bhaumik S, et al. Cost-effectiveness of interventions to control cardiovascular diseases and diabetes mellitus in South Asia: a systematic review. BMJ Open 2018;8:e017809. doi:10.1136/ bmjopen-2017-017809

- Prepublication history and additional material for this paper are available online. To view these files, please visit the journal online (http://dx.doi. org/10.1136/bmjopen-2017017809).

Received 19 May 2017 Revised 28 December 2017 Accepted 16 February 2018

Check for updates

For numbered affiliations see end of article.

Correspondence to

Dr. Kavita Singh;

kavita@ccdcindia.org

\section{ABSTRACT}

Objectives More than $80 \%$ of cardiovascular diseases

(CVD) and diabetes mellitus (DM) burden now lies in low and middle-income countries. Hence, there is an urgent need to identify and implement the most cost-effective interventions, particularly in the resource-constraint South Asian settings. Thus, we aimed to systematically review the cost-effectiveness of individual-level, group-level and population-level interventions to control CVD and DM in South Asia.

Methods We searched 14 electronic databases up to August 2016. The search strategy consisted of terms related to 'economic evaluation', 'CVD', 'DM' and 'South Asia'. Per protocol two reviewers assessed the eligibility and methodological quality of studies using standard checklists, and extracted incremental cost-effectiveness ratios of interventions.

Results Of the 2949 identified studies, 42 met full inclusion criteria. Critical appraisal of studies revealed 15 excellent, 18 good and 9 poor quality studies. Most studies were from India $(n=37)$, followed by Bangladesh $(n=3)$, Pakistan $(n=2)$ and Bhutan $(n=1)$. The economic evaluations were based on observational studies $(n=9)$, randomised trials $(n=12)$ and decision models $(n=21)$. Together, these studies evaluated 301 policy or clinical interventions or combination of both. We found a large number of interventions were cost-effective aimed at primordial prevention (tobacco taxation, salt reduction legislation, food labelling and food advertising regulation), and primary and secondary prevention (multidrug therapy for CVD in high-risk group, lifestyle modification and metformin treatment for diabetes prevention, and screening for diabetes complications every $2-5$ years). Significant heterogeneity in analytical framework and outcome measures used in these studies restricted metaanalysis and direct ranking of the interventions by their degree of cost-effectiveness.

Conclusions The cost-effectiveness evidence for CVD and DM interventions in South Asia is growing, but most evidence is from India and limited to decision modelled outcomes. There is an urgent need for formal health technology assessment and policy evaluations in South Asia using local research data.

PROSPERO registration number CRD42013006479.

\section{Strengths and limitations of this study}

- This is the first systematic review to synthesise cost-effectiveness evidence on all types of interventions (policy, clinical or behavioural) to control cardiovascular diseases and diabetes mellitus in South Asia.

- This review used a rigorous and broad search strategy including a wide range of sources to ensure all published studies are included for review.

- This review used explicitly stated methods (protocol paper published) and standard checklists to assess methodological quality of studies.

- The search was confined to English language publications performed as of August 2016, and this review excluded unpublished and 'grey' literature domain as we wanted to include studies that have undergone peer review process.

- Significant heterogeneity in analytical framework and outcome measures used in these studies restricted meta-analysis and direct ranking of the interventions by their degree of cost-effectiveness.

\section{INTRODUCTION}

Evidence from randomised trials suggests that both pharmacological and non-pharmacological strategies are important in prevention and management of cardiovascular diseases (CVD) and diabetes mellitus (DM) ${ }^{1-12}$ While there is strong evidence on cost-effectiveness of pharmaceutical and lifestyle interventions in reducing the CVD and DM risk in affluent settings, ${ }^{13-16}$ little is known about the comparative cost-effectiveness of various interventions to control CVD and DM in South Asia. To generalise results from high-income countries to low and middle-income countries (LMICs) is not entirely justified because reasonable thresholds for cost-effectiveness will vary markedly—as will affordability. Also, setting specific cost-effectiveness information 
is important because of the differences in healthcare infrastructure.

With the rapidly increasing prevalence of CVD and DM in South Asia and the consequent huge economic losses, coupled with ill-equipped health systems and scarce resources to tackle the burden of chronic conditions, it is imperative to promote the most cost-effective interventions in this region. While a large number of economic evaluations have been recently performed in context to LMICs, and some authors have reviewed the available literature on non-communicable diseases broadly, ${ }^{1718}$ no systematic attempt has been made so far to compile the evidence base and appraise the methodological quality of the economic evaluations of interventions to control CVD and DM in South Asia. To the best of our knowledge, no review has considered the cost-effectiveness evidence of interventions to control CVD and DM simultaneously, although these diseases share common risk factors.

We systematically reviewed the cost-effectiveness evidence on individual-level, group-level and population-level interventions to control CVD and DM in South Asia. The specific objectives were the following:

1. to summarise the incremental resource use, costs, consequences and cost-effectiveness of interventions versus comparators to control CVD and DM in South Asia

2. to describe the quality of economic evaluations considering key methodological issues.

\section{Research design and methods}

A protocol for the systematic review has been published previously and it provides a detailed description of the methodology, used for the current study. ${ }^{19}$ The systematic review has been registered previously in PROSPERO (CRD42013006479).

Briefly, we searched for studies that met the following inclusion criteria:

1. type of studies: full economic evaluations (cost-effectiveness analysis, cost-utility analysis, cost-benefit analysis) based on randomised trials or observational studies or decision models

2. type of participants: studies that included individuals with either established DM or CVD or at risk of developing these diseases in one of the South Asian countries: Afghanistan, Bangladesh, Bhutan, India, Pakistan, Maldives, Nepal and Sri Lanka

3. types of interventions: interventions or strategies for prevention and treatment of CVD or DM as documented in the previously published protocol ${ }^{19}$

4. types of outcome measures: we included several outcomes broadly under three domains-resource use, costs and cost-effectiveness as incremental cost per quality-adjusted life years (QALYs) gained, or disability-adjusted life years (DALYs) averted, or life years gained or intermediate outcomes; a detailed list has been presented in the previously published protocol $^{19}$

5. studies published in the English language.
We searched 14 electronic databases and hand-searched for publications of the Disease Control Priorities Project 2 (DCPP2) and the WHO-Choosing Interventions that are Cost-Effective (WHO-CHOICE) to identify relevant studies. The details of the databases searched and a search strategy are provided in supplementary web appendix 1 .

\section{Critical appraisal of included studies}

Checklists proposed by Drummond $e t a l,{ }^{20}$ Evers $e t a l^{21}$ and Philips $e t a t^{22}$ were used for data extraction and to review methodological quality and strength of economic evidence. Also, we looked for funding sources of included studies.

\section{Analysing, interpreting and reporting results}

We extracted the incremental cost, incremental effect and incremental cost-effectiveness ratios (ICER) for interventions evaluated in the eligible studies. To adjust for cost and varying currencies over time, we used country-specific consumer price inflation rate to present value in 2017 and then used midyear currency conversion. ${ }^{23} 24$ All costs were converted to US\$ (2017). Data extraction and critical appraisal of included studies were conducted by two authors independently and differences if any were resolved by consensus.

We used country-specific gross domestic product (GDP) per capita threshold, as per WHO guidelines, ${ }^{25}$ to interpret the ICER for all interventions evaluated in this review. We colour-coded ICER estimates as per the following scheme:

- green=ICER $<1 \times$ GDP per capita per QALY gained (highly cost-effective)

- yellow=1-3×GDP per capita per QALY gained (cost-effective)

- red=ICER $>3 \times$ GDP per capita per QALY gained (not cost-effective).

Interventions that resulted in a negative incremental effect were regarded as dominated strategy and no ICER was reported. Further, we synthesised the cost-effectiveness data and presented the ICER for policy or clinical interventions, separately in the following categories: primordial, primary, secondary and tertiary prevention.

\section{Difference between protocol and full review}

We have not planned to include economic evaluations based on observational studies in the protocol but we have included it in our review. The more inclusive criteria enabled us to provide a more comprehensive review of the evidence base surrounding the topic. Risk of bias assessment in randomised trials was not conducted using Cochrane methods as Drummond and Evers checklists are inclusive of methodological quality assessments of economic evaluations alongside randomised trials as well.

\section{RESULTS}

\section{Search results}

Our first search yielded 2949 items, titles and abstracts screening resulted in 85 articles, and full-text screening provided 42 articles that met full inclusion criteria (figure 1). 

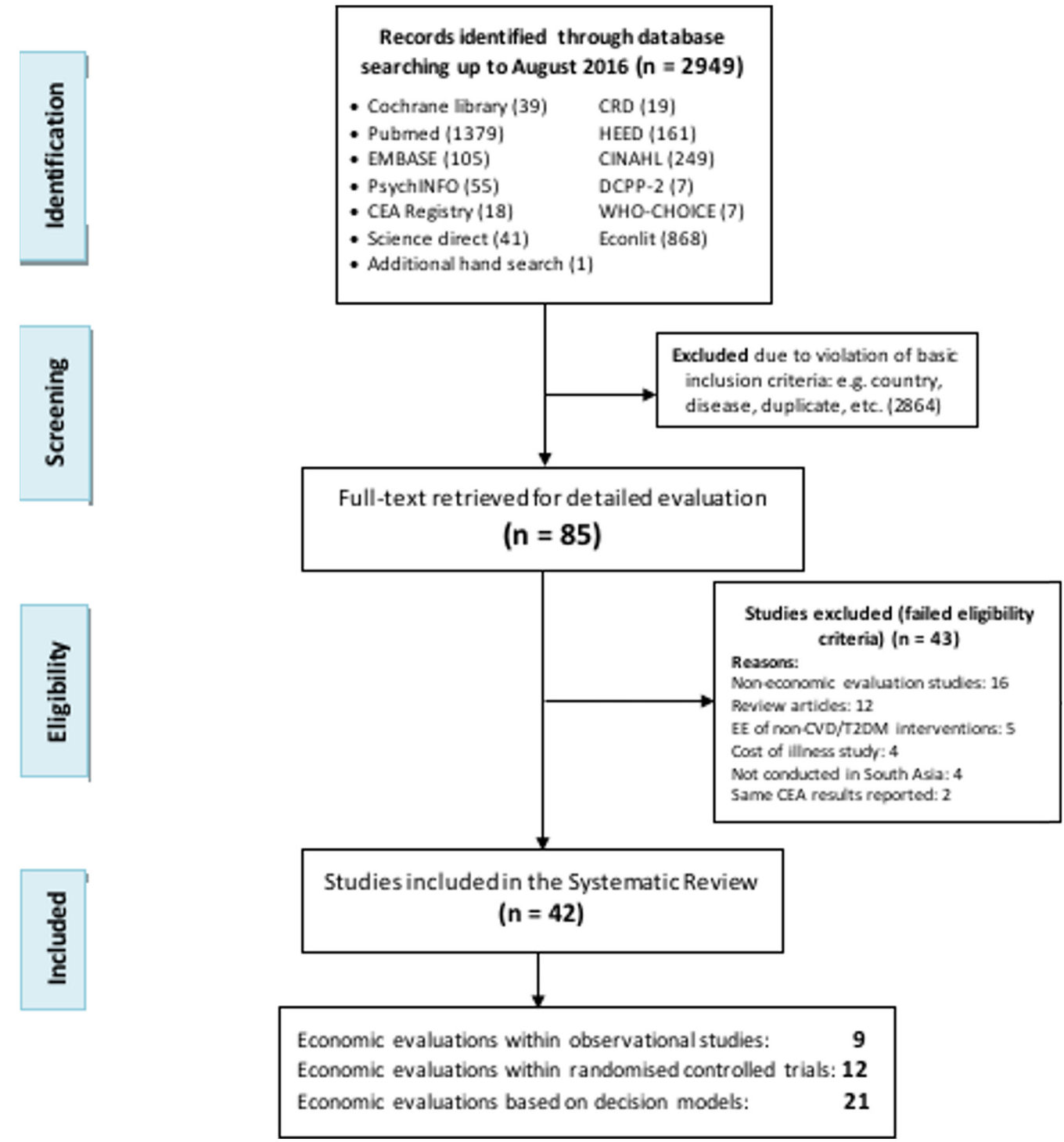

Figure 1 PRISMA flow chart for the selection of economic evaluations of interventions to control cardiovascular disease and diabetes mellitus in South Asia. CEA, cost-effectiveness analysis; CINAHL, Cumulative Index to Nursing and Allied Health Literature; CRD, Centre for Reviews and Dissemination; CVD, cardiovascular disease; DCPP2, Disease Control Priorities Project 2; EE, economic evaluation; HEED, Health Economic Evaluation Database; PRISMA, Preferred Reporting Items for Systematic Reviews and Meta-Analyses; T2DM, type 2 diabetes mellitus; WHO-CHOICE, WHO-Choosing Interventions that are CostEffective.

\section{Characteristics of included studies}

Table 1 shows the detailed description of the studies $(\mathrm{n}=42)$ by country/setting, study population, intervention(s), comparator(s), economic perspective and type of analysis, and outcome measures.

\section{Study design}

The economic evaluations were based on observational studies $(n=9)$, randomised controlled trials (RCT) $(n=12)$ and decision models $(\mathrm{n}=21)$.

\section{Study setting}

Most studies were from India ( $\mathrm{n}=37$ ), followed by Bangladesh $(n=3)$, Pakistan $(n=2)$ and Bhutan $(n=1)$. Decision modelling studies had used effectiveness data mostly from meta-analysis of RCTs that reported results from developed countries.

Study population

Individuals (or population) at risk or with established CVD or DM were included.

\section{Intervention targets and comparators}

Three hundred and one interventions (policy, clinical or behavioural) were evaluated against null scenario (no intervention) or active comparators.

\section{Perspective}

In two-thirds of the studies $(\mathrm{n}=28)$, the authors explicitly documented and justified the economic perspective of the study. The studies used 'health system', that is, direct 


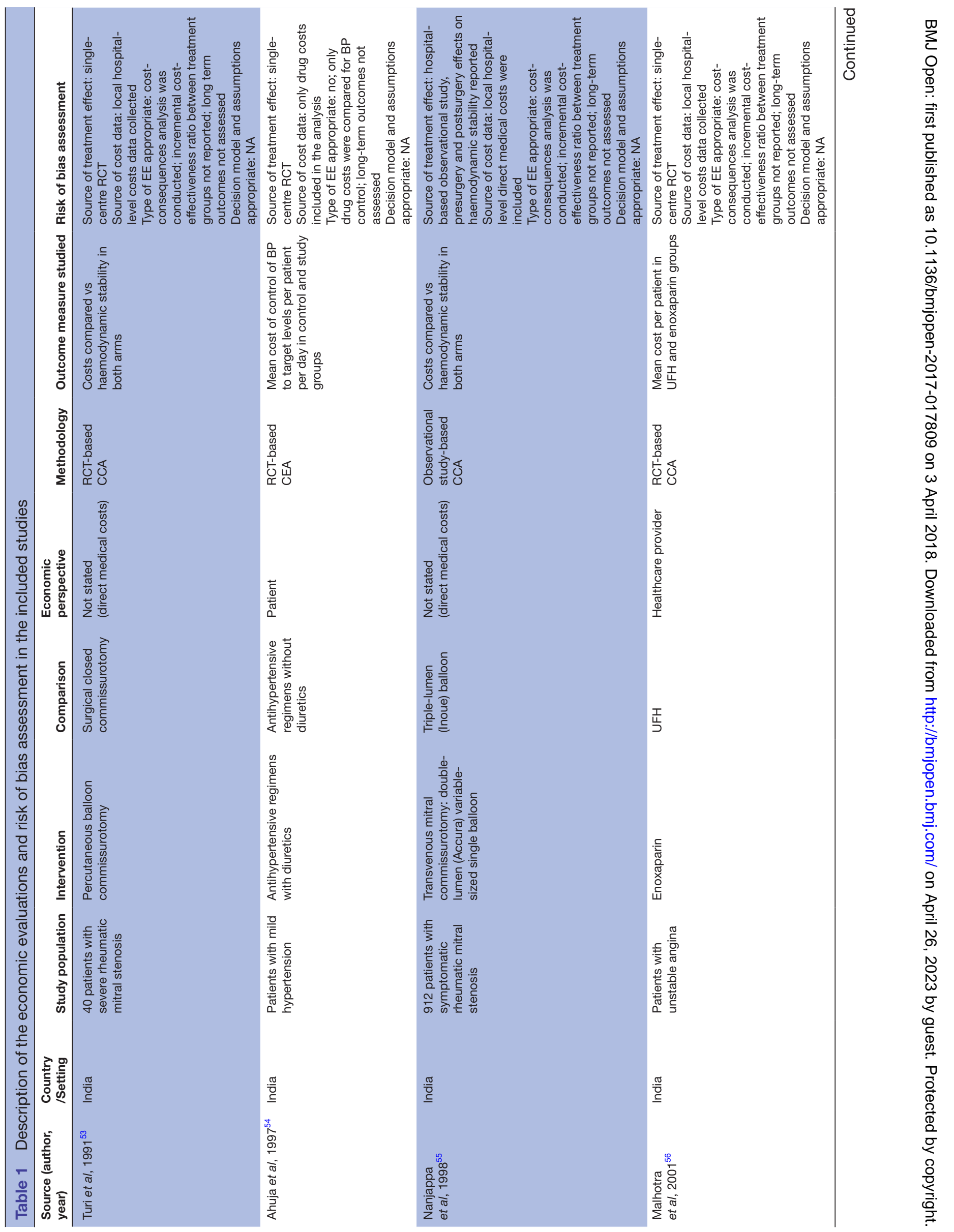




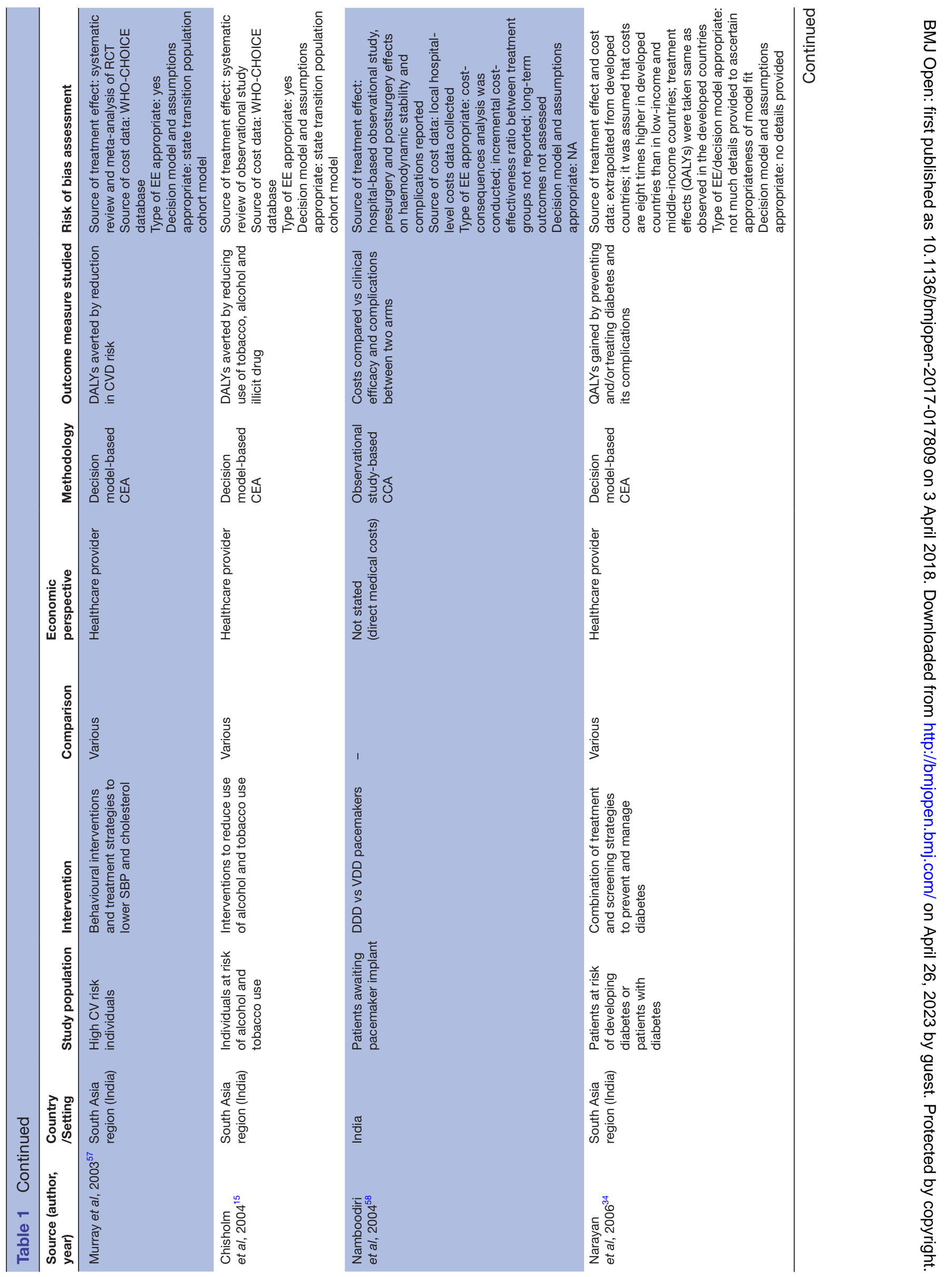




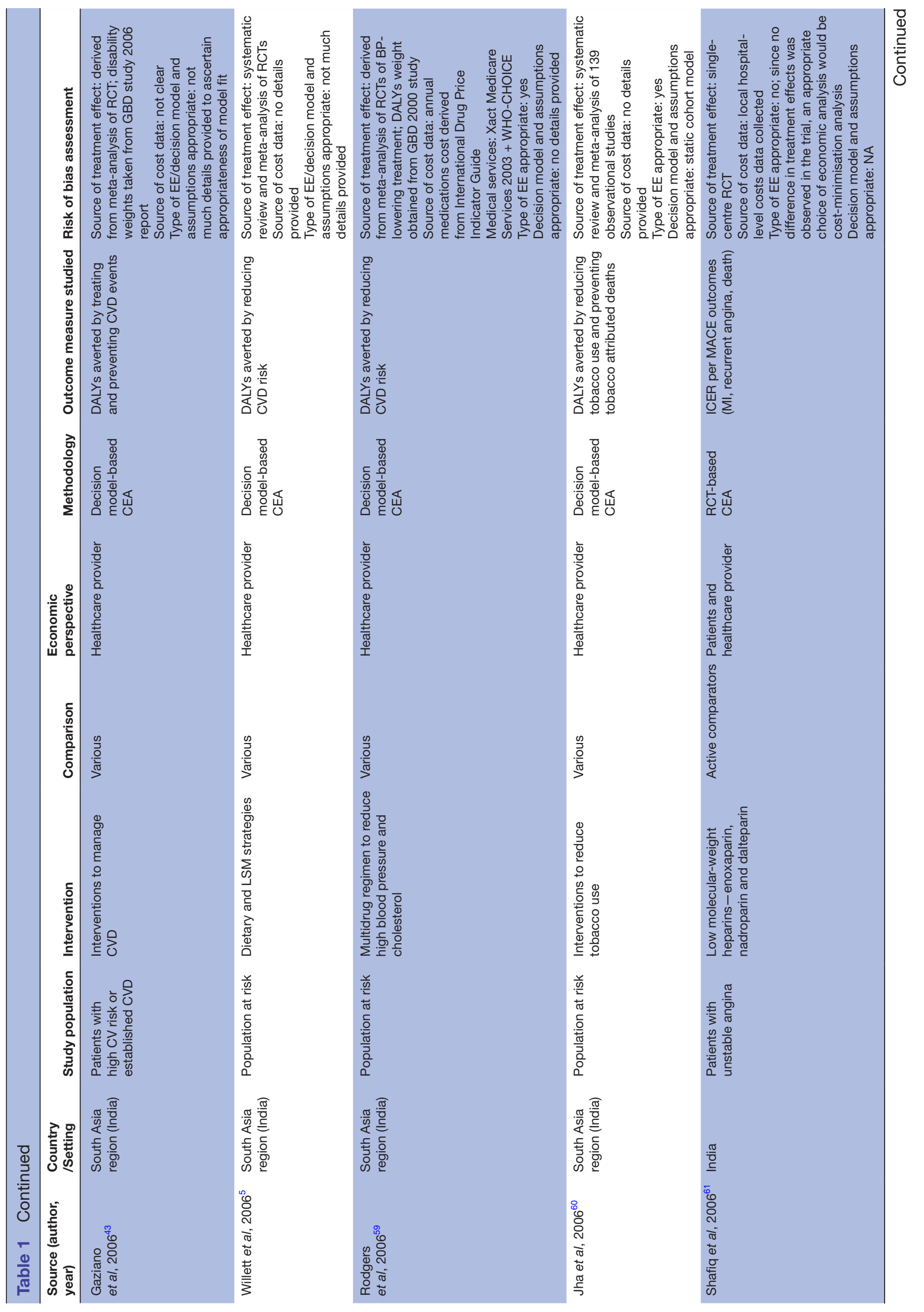

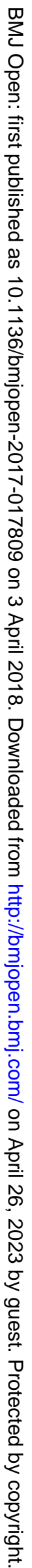




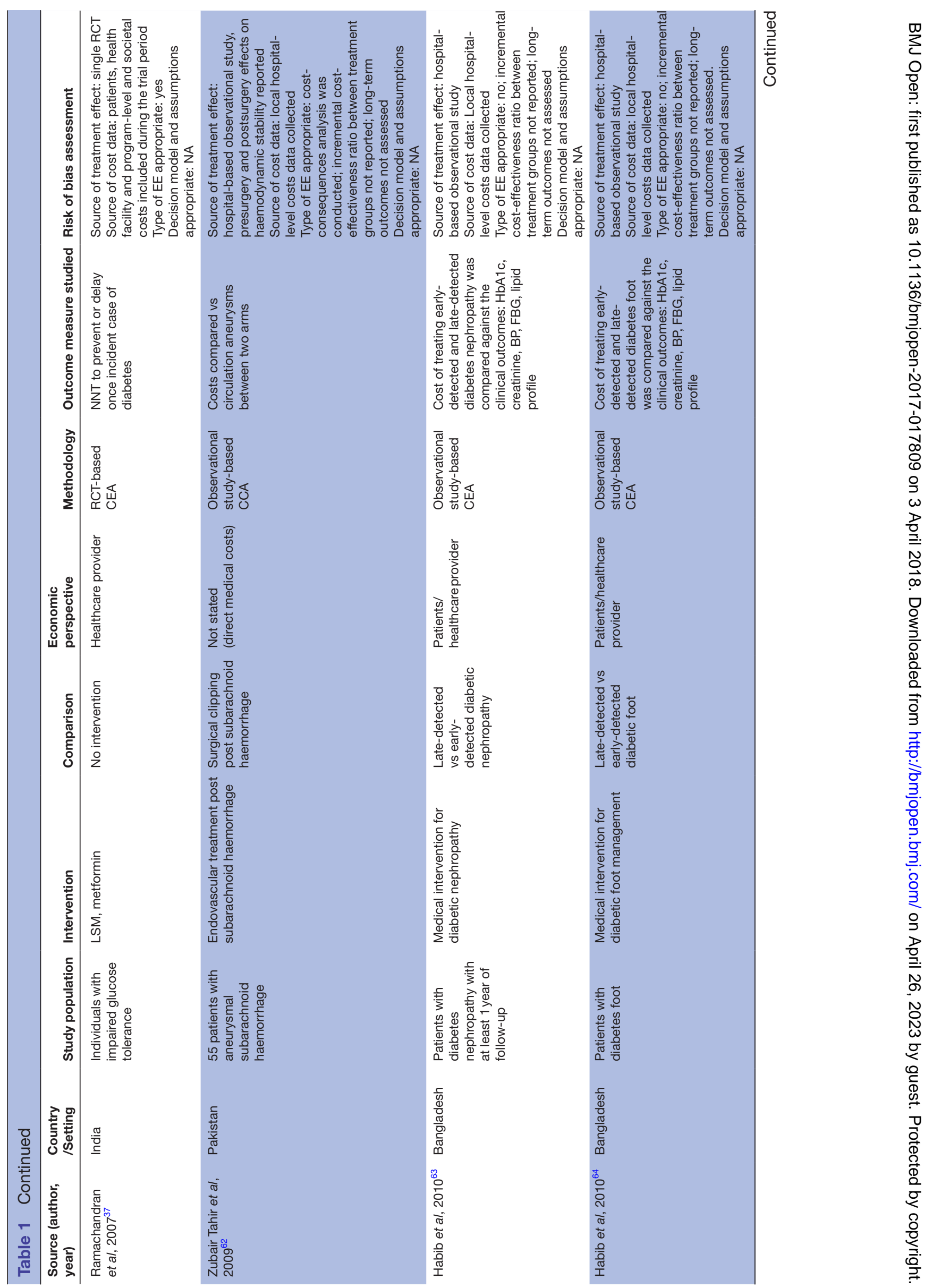




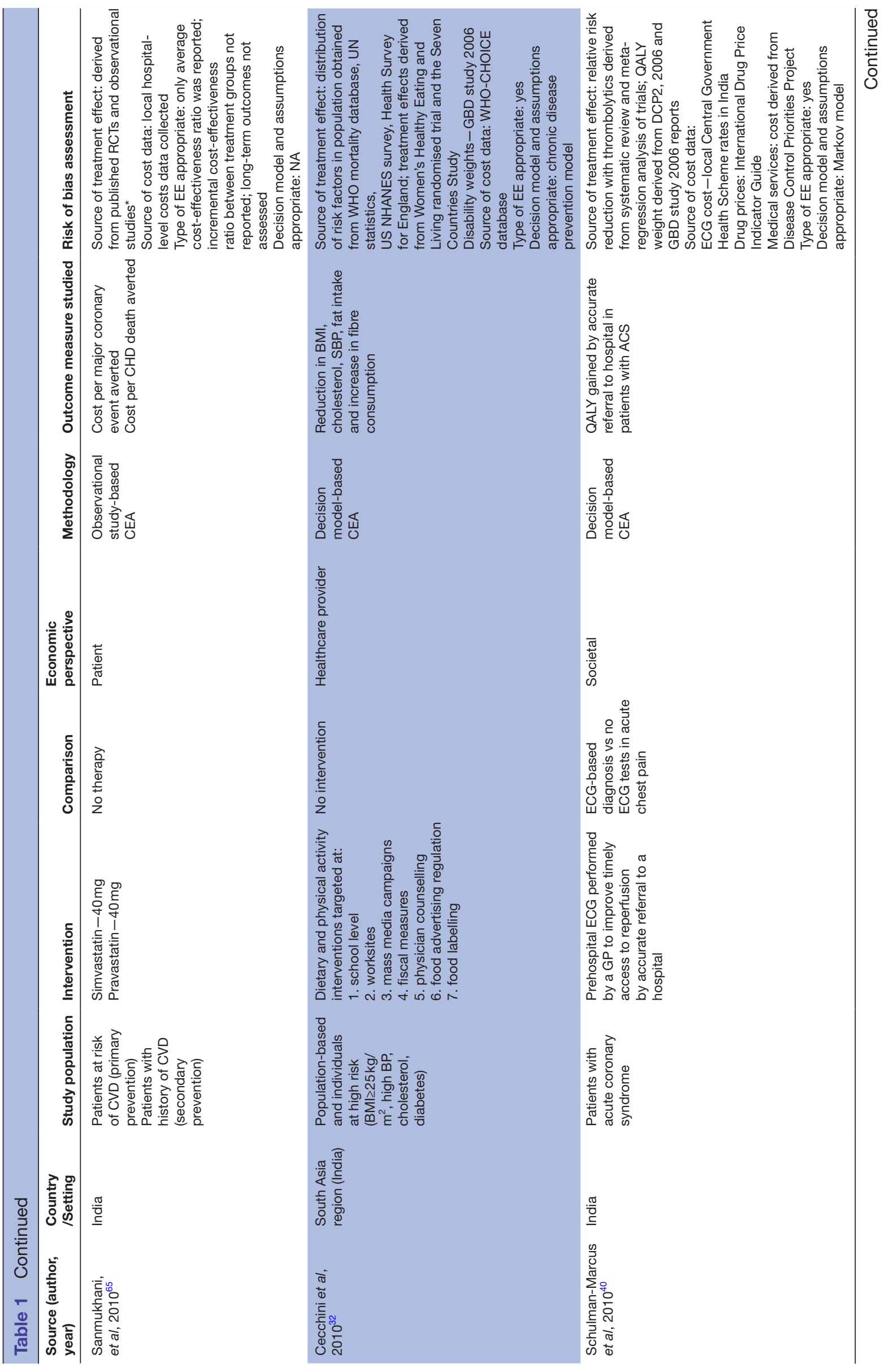




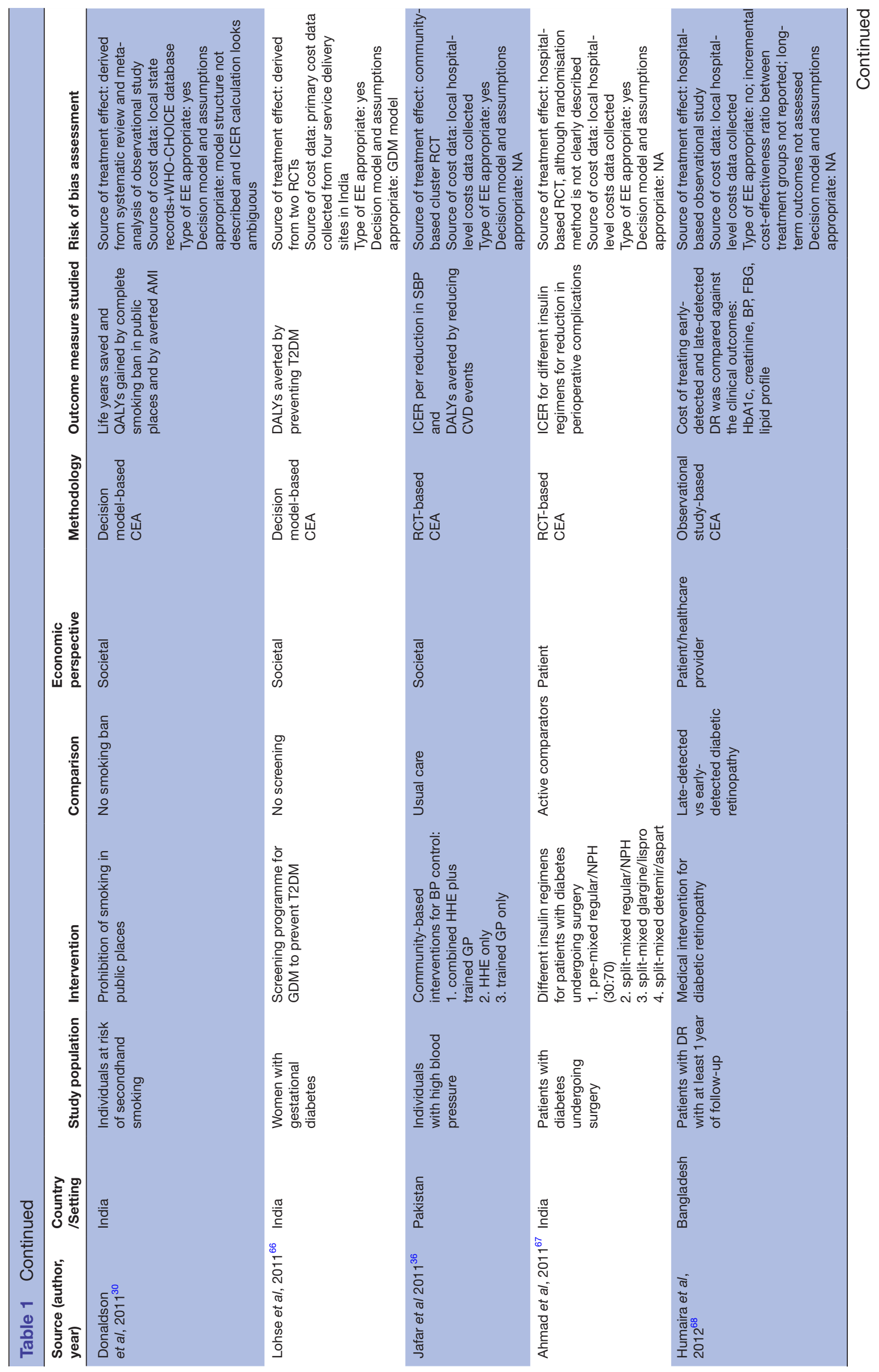

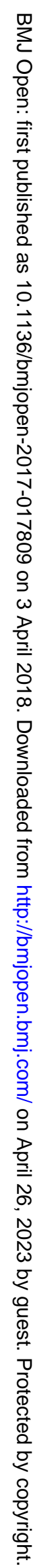




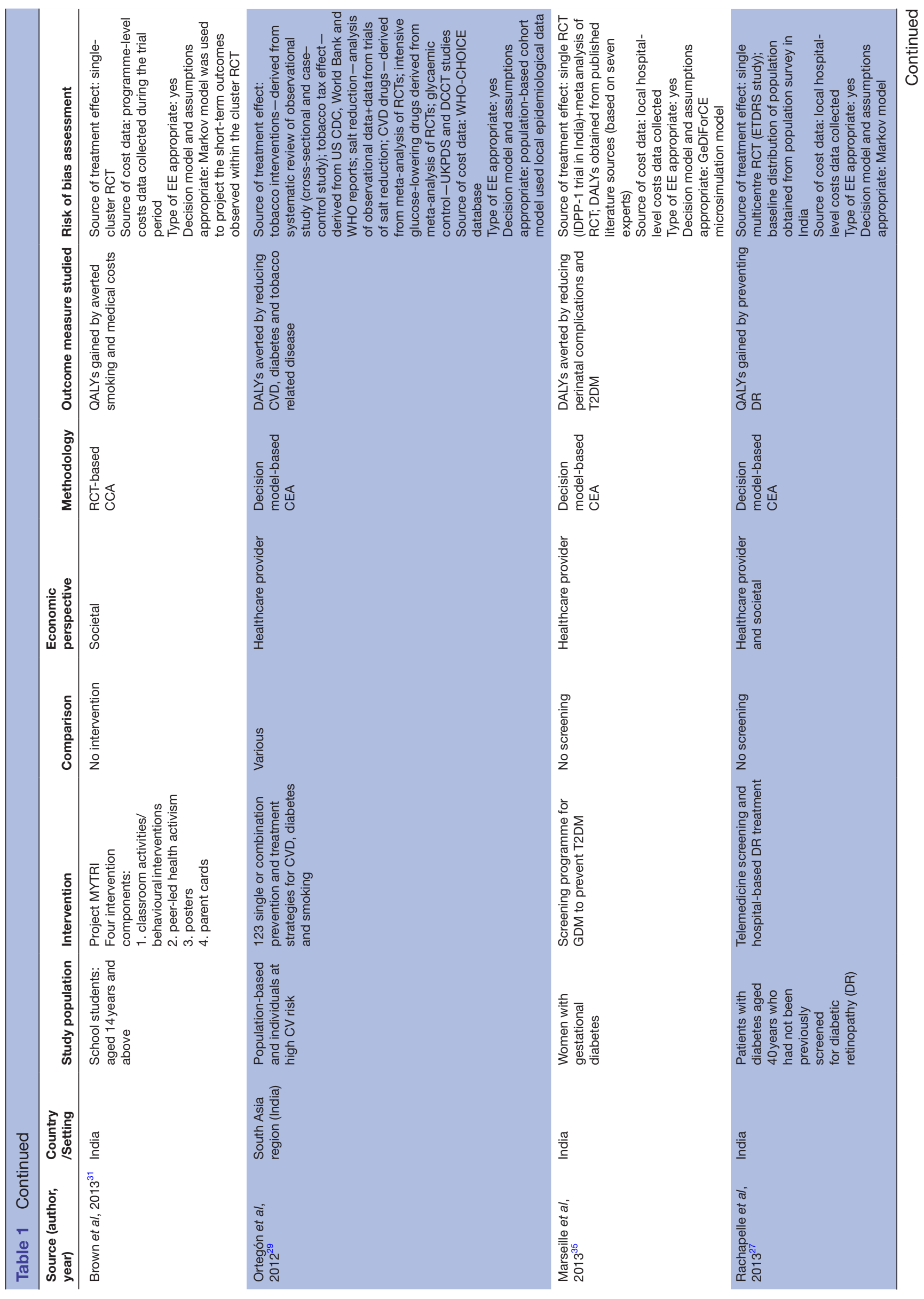




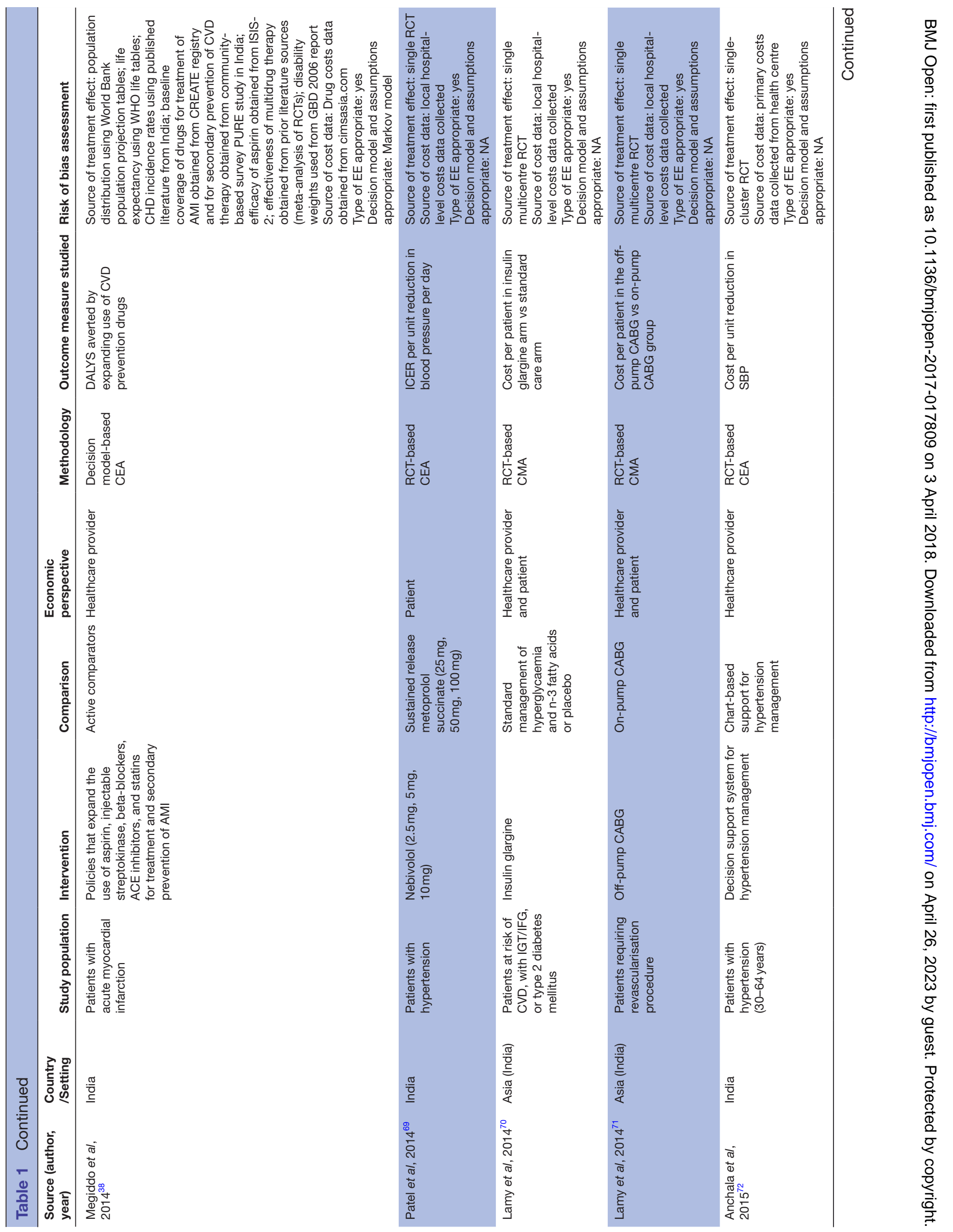




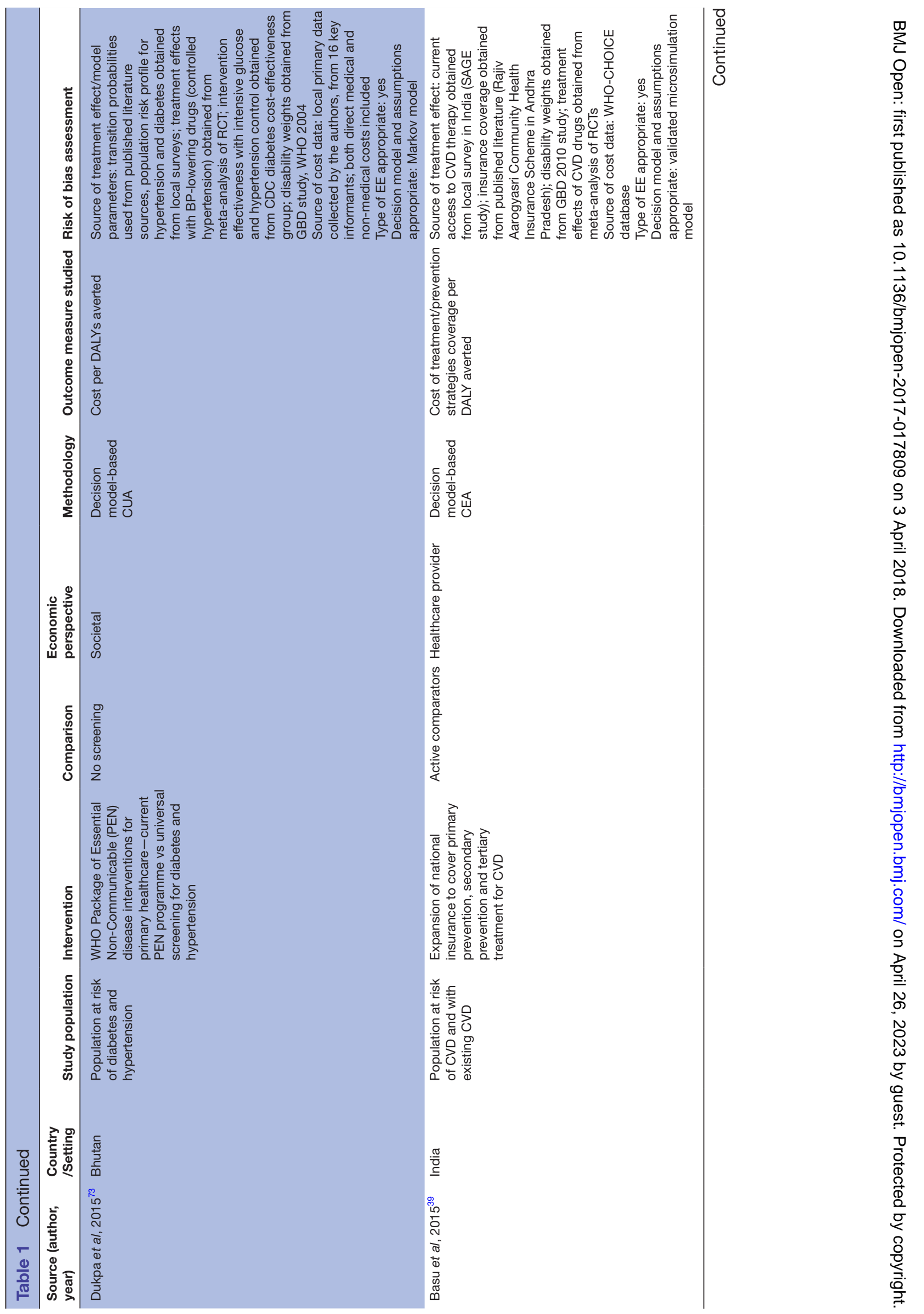






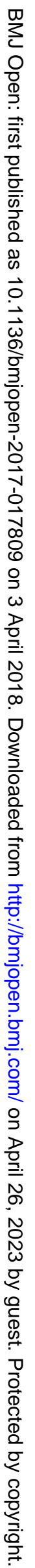




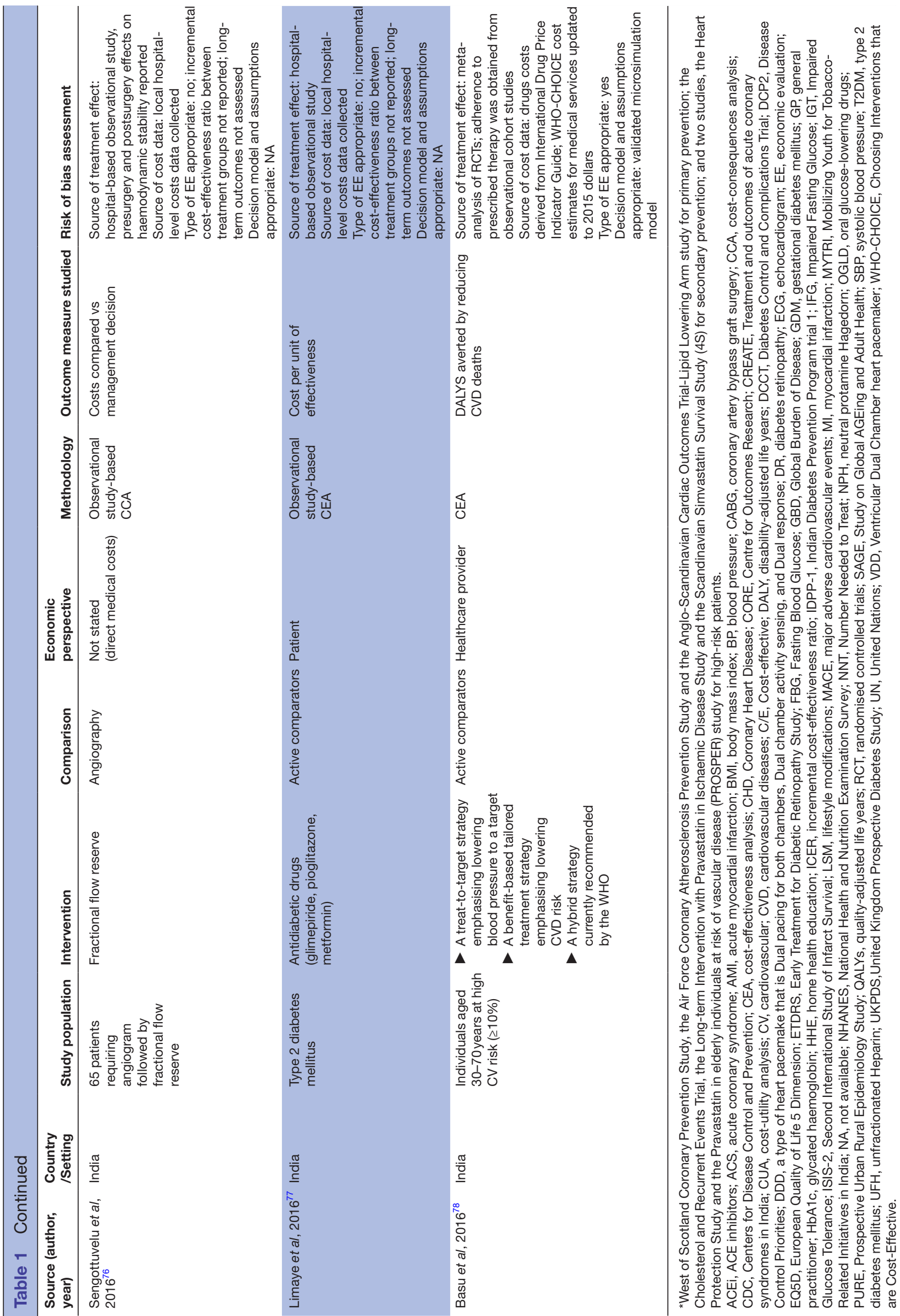


costs incurred by the health system $(\mathrm{n}=26)$; 'patient', that is, out-of-pocket payments by patient $(\mathrm{n}=6)$; or 'societal', that is, inclusive of all direct and indirect costs, plus productive loss $(n=6)$ perspectives. Five studies did not state any perspective.

\section{Funding}

Two-thirds of evaluations ( $\mathrm{n}=29)$ provided statements on the funding source. Public sponsorship or charitable trust/foundation grant was most common $(n=16)$, followed by pharmaceutical industry $(n=6)$ or received no support $(n=7)$. A large number of studies did not state their source of research funding $(n=13)$.

\section{Resource use and costs}

Only $20 \%$ of the studies $(n=8)$ reported types and quantities of resource use and unit costs separately. Of these, five were RCT-based economic evaluations and two were decision model studies, suggesting that RCT provides an advantage on the reporting of actual resource use data as it is being collected during the trial.

Mostly direct medical costs were considered, although the scope of this varied enormously. For instance, 14 studies included only cost of intervention (medicines, diagnostics), while others $(n=28)$ included cost of training, delivery of intervention, associated healthcare visit costs and travel cost of patients to the healthcare facility. Most $(n=27)$ appeared to use an 'ingredients' costing approach, where costs were broken down between the main cost components such as medications, healthcare visits, vehicles, salaries and consumables. Fewer $(\mathrm{n}=5)$ used an 'activity'-based approach, by identifying specific tasks such as programme and therapy costs. Two studies appeared to use some combination of the two, and it was not possible to discern the approach for eight papers. Few studies $(n=6)$ also included 'productivity losses' (often termed 'indirect costs') in their assessment of costs, which were measured using the 'human capital approach'.

Regardless of the approach taken, most papers $(n=21)$ presented aggregated cost information. Many studies used actual expenditure data $(n=17)$ as their source of costs data. Seven studies used published sources to generate cost estimates sometimes supplemented with expert opinion. Currencies reported were mostly in US\$ $(n=25)$, international dollars $(n=4)$ or local currencies (Indian rupees/Bhutanese rupees) $(\mathrm{n}=6)$. In addition, seven studies quoted costs in both US\$ and the local currency.

\section{Outcome measures (consequences)}

Nearly half of the studies $(n=21)$ used 'life years gained' or 'QALYs' or 'DALYs' in their analysis. The calculation of QALYs/DALYs was based on South Asian population life expectancies; however, the utility values (QALYs weight) were derived from developed countries. Disability weights used in the WHO-CHOICE-based decision model studies were derived from the Global Burden of Disease (GBD) study (2000).$^{26}$ The remaining studies reported intermediate outcome measures such as number needed to treat, length of hospital stay, hospitalisation rate, blood pressure (BP) reduction or CVD events avoided, which are easier to measure but harder to compare across interventions. None of the studies expressed outcomes (benefits) in monetary units.

\section{Time horizon}

Three-fourths of studies $(n=31)$ explicitly stated their analytical time horizon. Eighty per cent of decision model studies adopted lifetime horizon and others reported cost-effectiveness estimates for 10, 20, 25, 30 or 50 years. RCT/observational studies-based economic evaluations had a median time horizon of 1 year.

\section{Discounting}

A discount rate of $3 \%$ was most often used for both costs and effects in decision model studies. RCT-based economic evaluations used a discount rate of $3 \% \quad(n=3)$ and 5\% (n=1). Further, 11 studies did not apply any discount rate.

\section{Analytical approach}

Cost-effectiveness analysis or cost-utility analysis were the main methods $(n=34)$, followed by cost-consequences analysis $(n=6)$ and cost-minimisation analysis $(n=2)$. Although several of these papers $(n=8)$ described themselves as cost-effectiveness analysis, they were in fact cost-consequences analysis or cost-minimisation analysis because an incremental analysis was not reported or there was no significant difference in the effectiveness of the intervention versus comparator, respectively. Most studies reported average cost-effectiveness ratio and interpreted it as ICER against the comparator as null scenario, that is, no intervention.

We found several different types of decision models used for cost-effectiveness analysis. A large majority of the studies used the WHO-CHOICE state transition model. Others used coronary heart disease (CHD) policy model, GeDiForCE, IMS Centre for Outcomes Research Diabetes Model, Centers for Disease Control and Prevention (CDC) model, Markov model or individual microsimulation model. Few studies provided details of model validation.

\section{Sensitivity analyses and generalisability of study results}

Nearly half of the studies $(n=25)$ undertook some form of sensitivity analysis to assess the robustness of their findings to assumptions about input parameters. Of these, one-way sensitivity analysis was most often applied. Two studies used threshold analysis and one performed a multi-way sensitivity analysis. None considered the structural variations in the decision model for sensitivity analysis. Few studies described the model validation methods.

Three-quarters of the studies $(n=32)$ discussed the generalisability issue. Efforts were largely confined to stating the limitations of the study, such as whether randomisation was employed or noting one or two facts 
about the study site which might limit generalisability to other contexts. Another 12 studies discussed issues of affordability but in brief terms, for example, by noting that the available budget should be taken into account (most studies focused on the cost-effectiveness without considering the budget impact/constraint) or by questioning the sustainability of a novel service such as a mobile diabetic retinopathy services, where there are already existing health services. ${ }^{27}$

\section{Risk of bias assessment}

In our critical review of methods used in economic evaluations to assess risk of bias, we found that almost all economic evaluations based on observational study only presented costs and consequences of two treatment strategies separately, without reporting an ICER or employed sensitivity analysis to assess robustness of costs or treatment effect estimates. Also, estimates of treatment effects from the observational studies are not very reliable due to the limitations in the original study design. On the other hand, economic evaluations based on RCTs reported better economic outcomes, that is, ICERs; however, these studies were limited by short follow-up duration (30 days to 1 or 2 years), treatment effects assessed as intermediate clinical outcomes (BP reduction, number needed to prevent one DM case) and mostly direct medical costs from health system perspective or patient perspective were reported, which ignores the societal costs and productivity loss due to illness. Lastly, decision modelling studies reported ICER per QALY gained or DALY averted mostly using the WHO-CHOICE methods, Markov models or microsimulation models from societal or health system perspectives. Many of the decision model studies from DCPP did not report the source of costs data, source of QALY weights and details on decision model structure or validation methods. Further, most of the WHO-CHOICE-based generalised cost-effectiveness analysis used disability weights from an earlier version of the GBD study (2000). Therefore, findings from this review should be used with caution for local decision making, and there is an urgent need for more investment in local research to generate evidence/data on costs of treatment and health services and effectiveness of interventions (table 1).

\section{Methodological quality: summary}

Figures 2 and 3 report the overall quality of studies based on the key methodological issues and technical characteristics for decision model studies, respectively. In general, very few studies reported quantities of resource use data and unit costs separately, details of statistical tests used and CI around ICER estimates. Among decision model studies, none reported methods used to assess methodological, structural or heterogeneity uncertainties, and very few discussed model validation methods. Critical appraisal of studies revealed that there were 15 excellent $(++), 18 \operatorname{good}(+)$ and 9 poor quality studies (-) (table 2).

\section{Methodological quality of included studies ( $n=42)$}

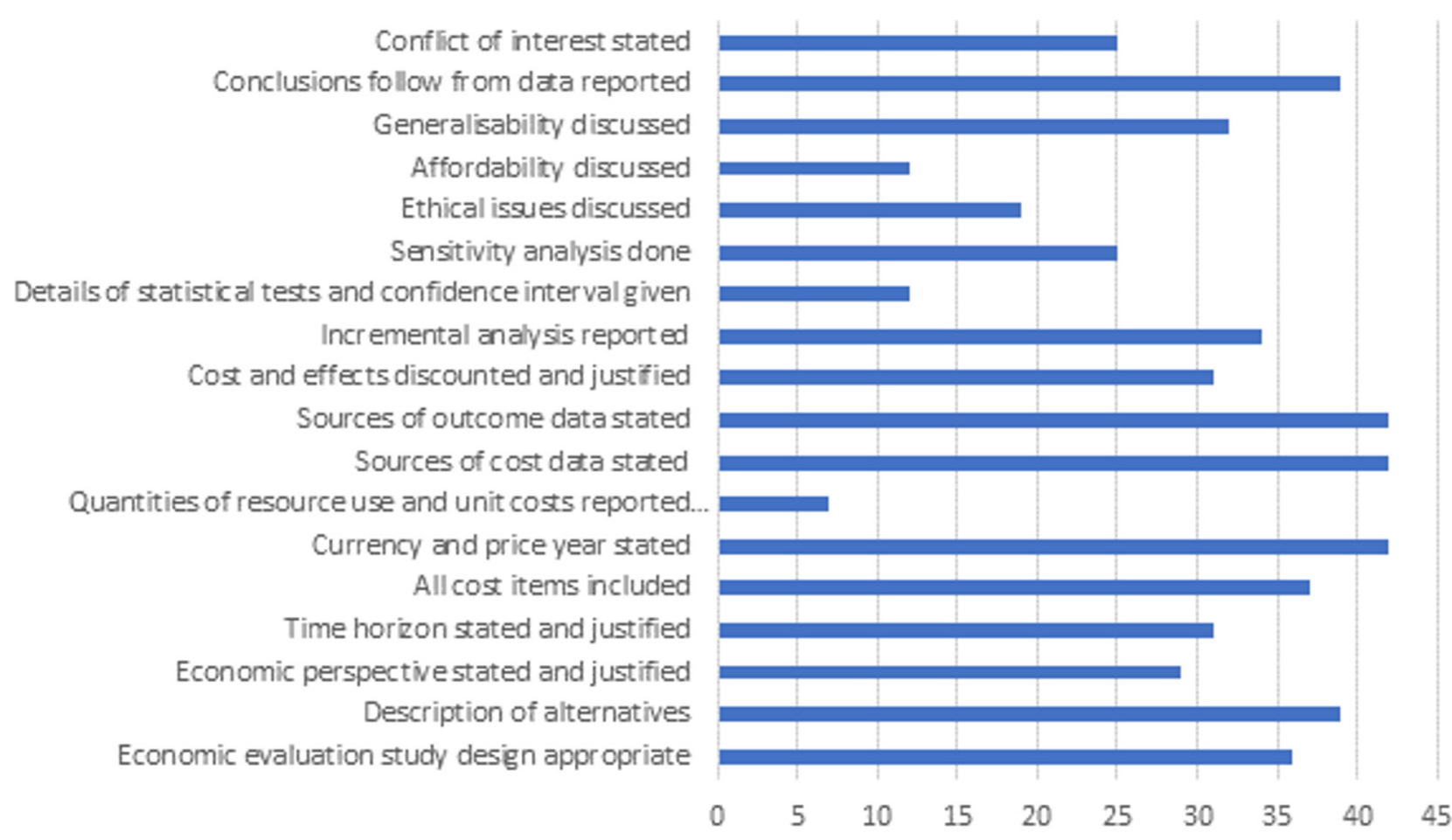

Figure 2 Methodological quality of included studies. This figure presents the number of studies meeting the key methodological quality metrics of economic evaluations as recommended in the standard checklists. 


\title{
Technical characteristics in Decision modeling studies $(n=21)$
}

\author{
Model results compared with other modelling studies \\ Model validation performed using independent data \\ Methodo logical uncertainty \\ Structur al uncer tainty \\ Heter ogeneity uncer tainty \\ Parameter uncertainty \\ Assumptions for continuing effect of treament justified \\ Extrapolation of final outcomes justified \\ Transition probability calculated appropriateiy \\ Data identification methods appr opriate \\ Cycle length stated and just fied \\ Chosen model type appropriate
}

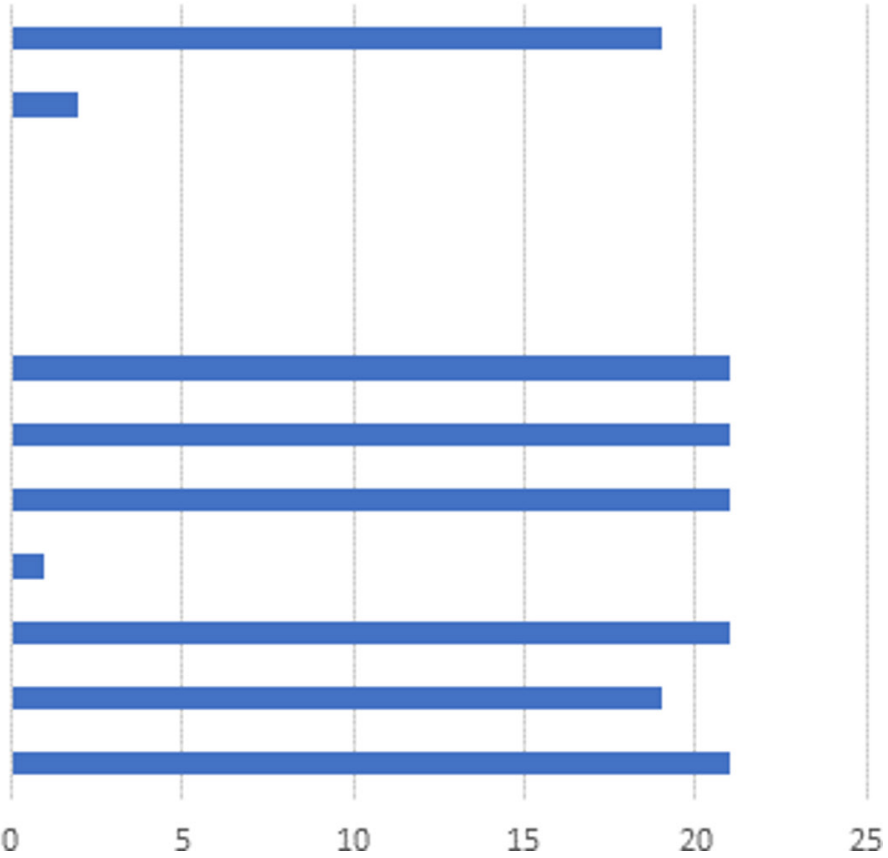

Figure 3 Technical characteristics of decision modelling studies. This figure presents the number of decision modelling studies meeting the key methodological criteria for decision modelling studies as proposed by Philips et al. ${ }^{22}$

\section{Cost-effectiveness evidence}

Interventions reviewed for their cost-effectiveness are grouped under the scheme of primordial, primary, secondary and tertiary prevention of CVD and DM (table 3). This flow is used to make information available in an accessible format for policy-level and clinical decisions. Cost-effectiveness results from observational studies have not been included in the final synthesis of cost-effectiveness data from South Asia due to poor quality of evidence. Cost-effectiveness data presented below are for India unless otherwise specified (the GDP per capita (in US\$2016) for India, Pakistan and Bhutan are 1861.5, 1468.2 and 729.5 , respectively). ${ }^{28}$

\section{Primordial prevention}

We found that a multicomponent population-level policy intervention consisting of increase in tobacco tax, clean indoor air law, advertisement ban and information/labelling are all highly cost-effective than increased tobacco tax alone $(<1 \times$ GDP per capita per DALY averted). ${ }^{29}$ Addition of 'nicotine replacement therapy', 'brief advice' or 'physician counselling' to the combination strategy for tobacco control was not cost-effective ( $>3 \times$ GDP per capita per DALY averted) ${ }^{29}$ Complete smoking ban in public places is also highly cost-effective in terms of life years gained and acute myocardial infarction averted. ${ }^{30}$ School-based smoking prevention programme as evaluated in a cluster randomised trial in India ${ }^{31}$ was found to be cost-effective (1-3×GDP per capita per QALY gained). Salt reduction by legislation was cost-effective $(1-3 \times$ GDP per capita per DALY averted) ${ }^{29} 32$ Substitution of trans fat with polyunsaturated fatty acids was cost-effective compared with null scenario (no intervention) per DALY averted. ${ }^{32}$ Media campaign to reduce saturated fat content was also cost-effective per DALY averted. ${ }^{32}$ A combined intervention of salt reduction by means of legislation together with public education campaign is cost-effective too. ${ }^{32}$ Alcohol taxation combined with advertisement ban was the most cost-effective strategy for alcohol control. ${ }^{15}$

\section{Primary prevention}

A 2015 modelling study conducted in Bhutan demonstrated that universal screening for diabetes and hypertension was highly cost-effective compared with no screening $(<1 \times$ GDP per capita per QALY gained $) .{ }^{33}$ Another 2006 modelling study from India ${ }^{34}$ showed that screening undiagnosed diabetes and treating those who test positive were not cost-effective, with an ICER of US $\$ 11671$ per DALY averted (ie, $>3 \times$ GDP per capita for India), suggesting that screening for diabetes alone was not cost-effective and it should be supplemented with other risk factors, for example, hypertension. Other factors that could have influenced conflicting results include different health system-related cost, different model structure/model parameters, disease prevalence and time period.

Screening for gestational DM to prevent DM was also cost-effective compared with no screening. ${ }^{35}$

Among clinical interventions, preventive multidrug treatment provided to those at $>35 \%$ cardiovascular risk vs $5 \%$ cardiovascular risk over 10 years was more cost-effective. ${ }^{29}$ Combined strategy of home health education plus trained general physician for 


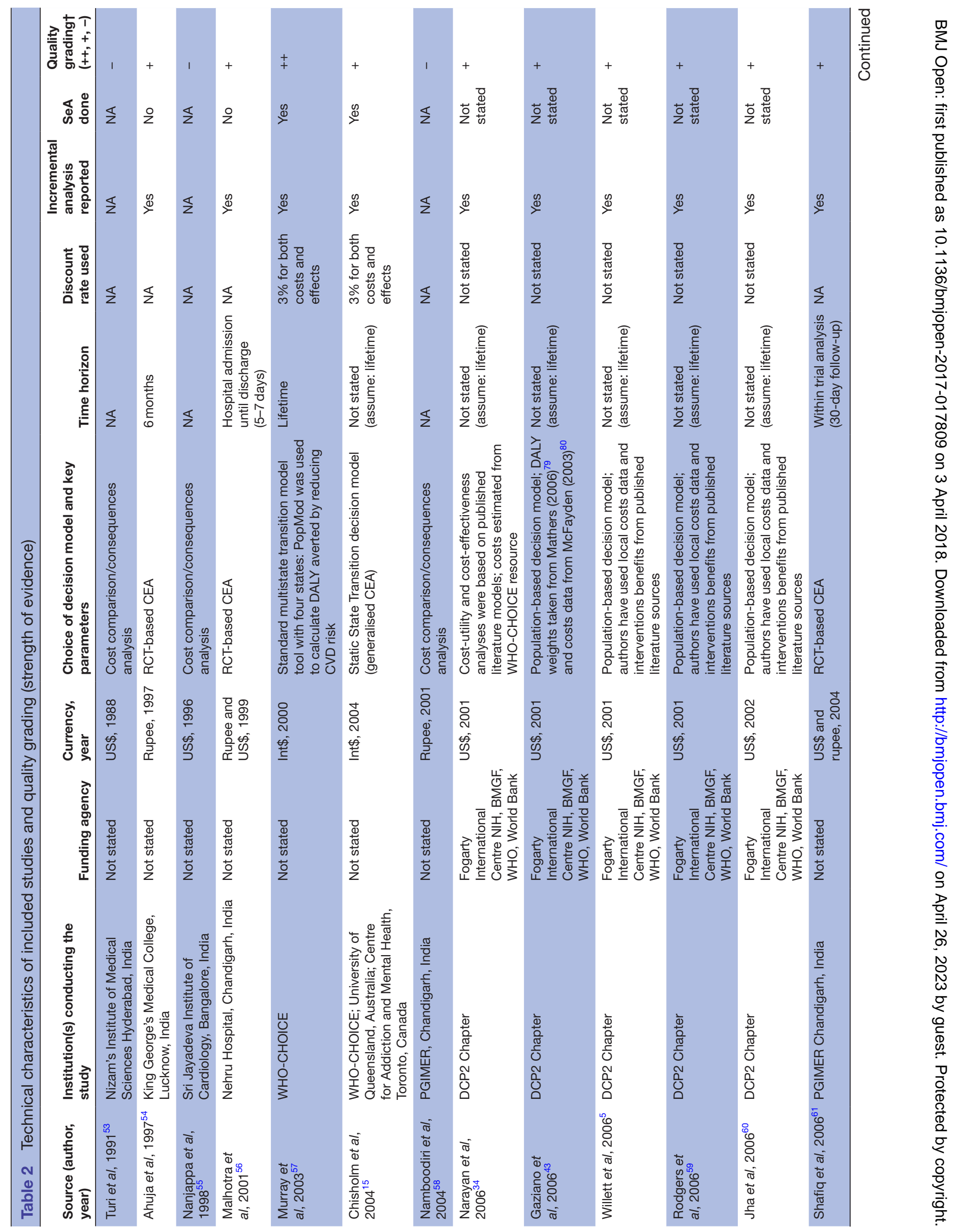




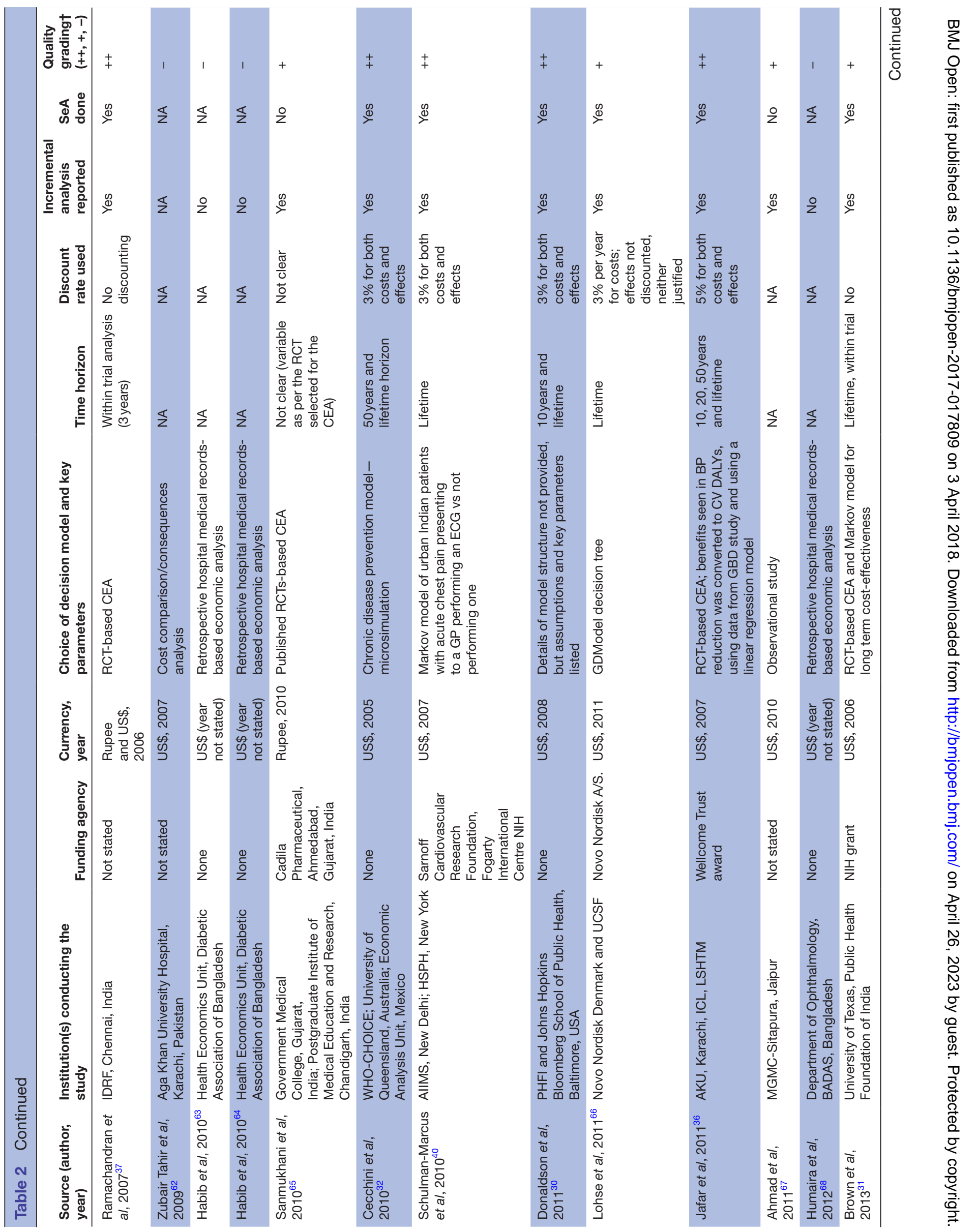




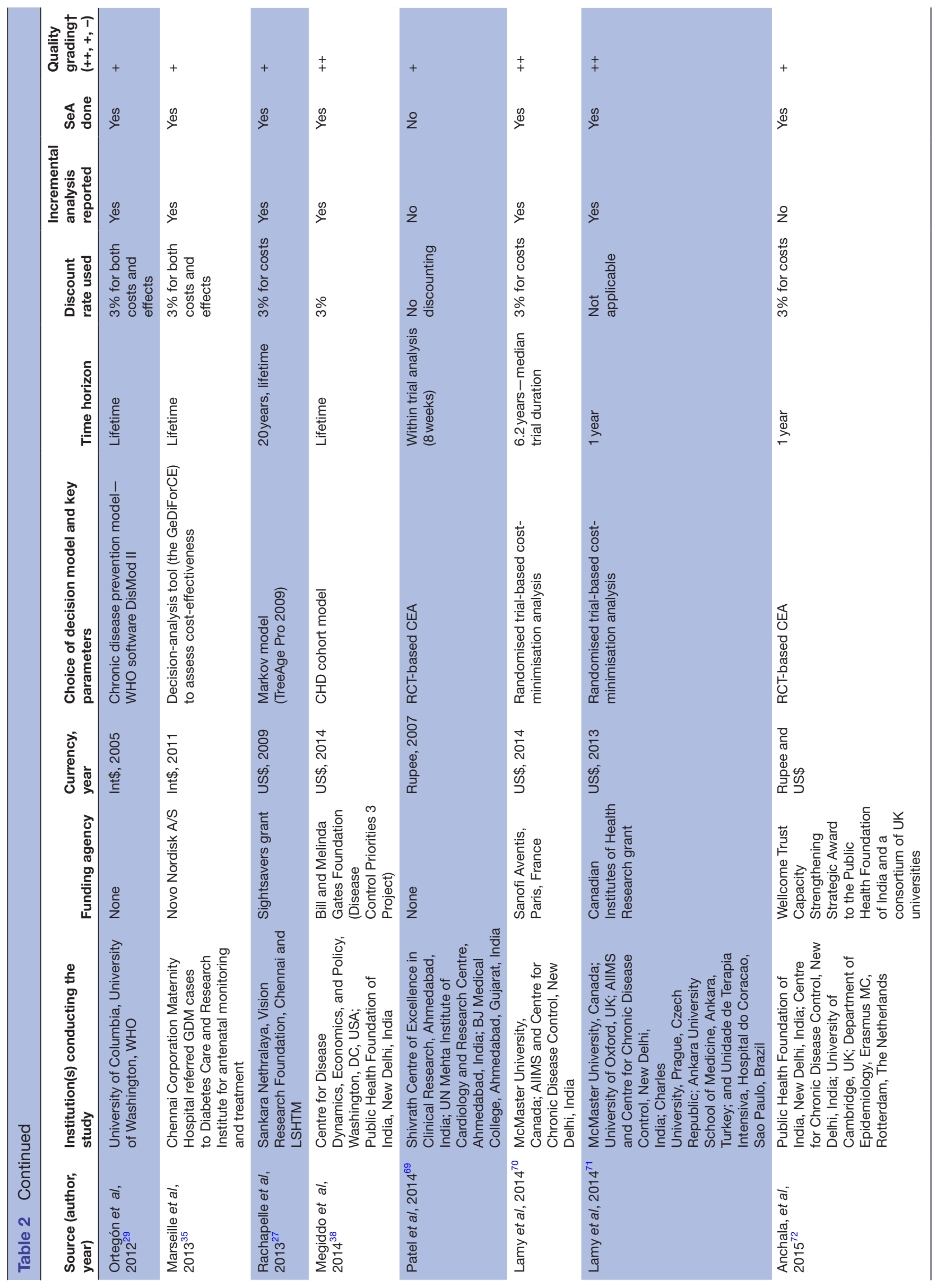




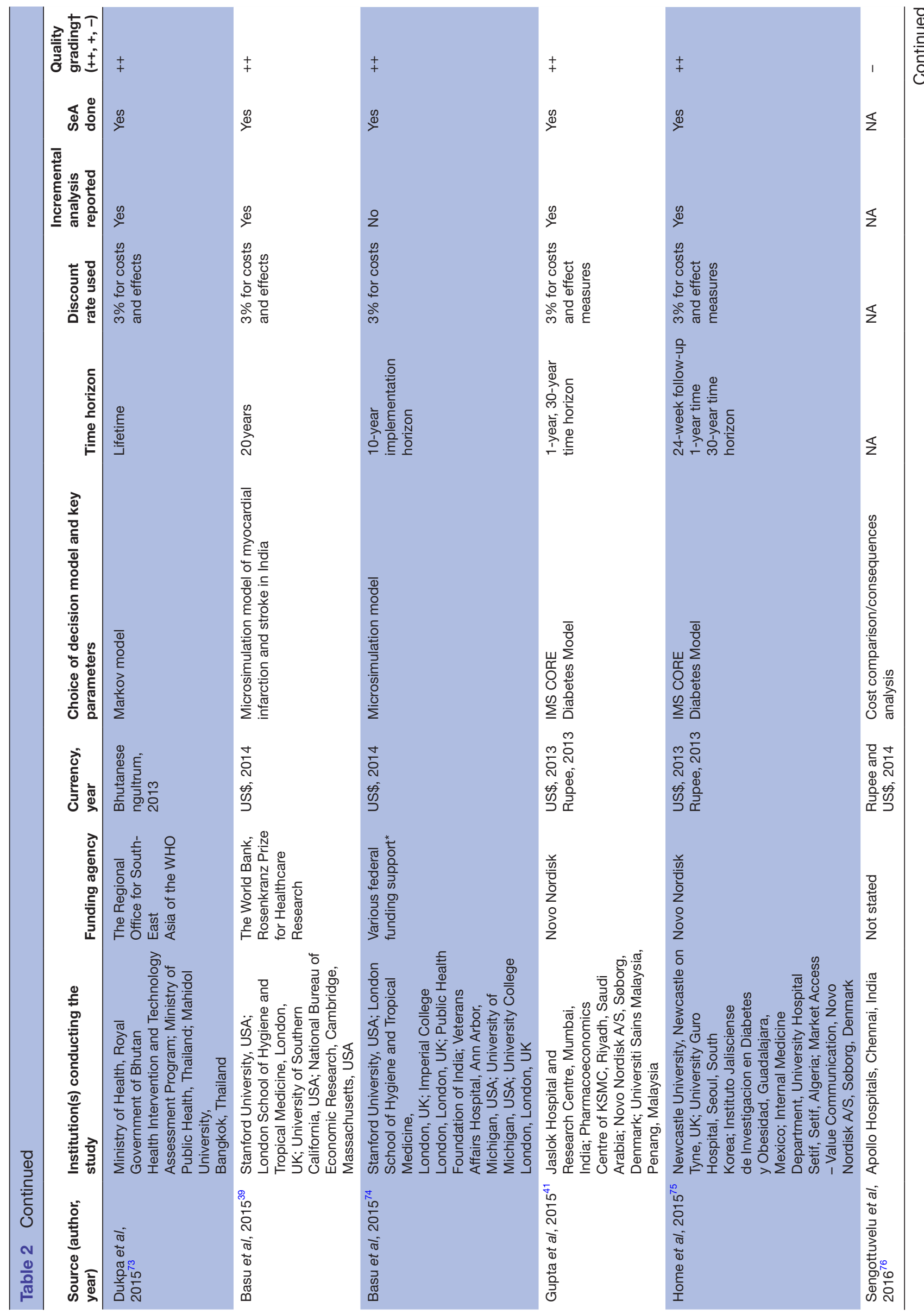

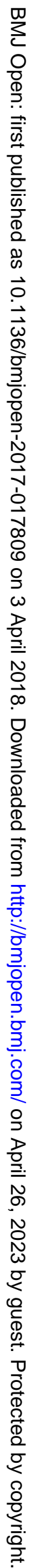




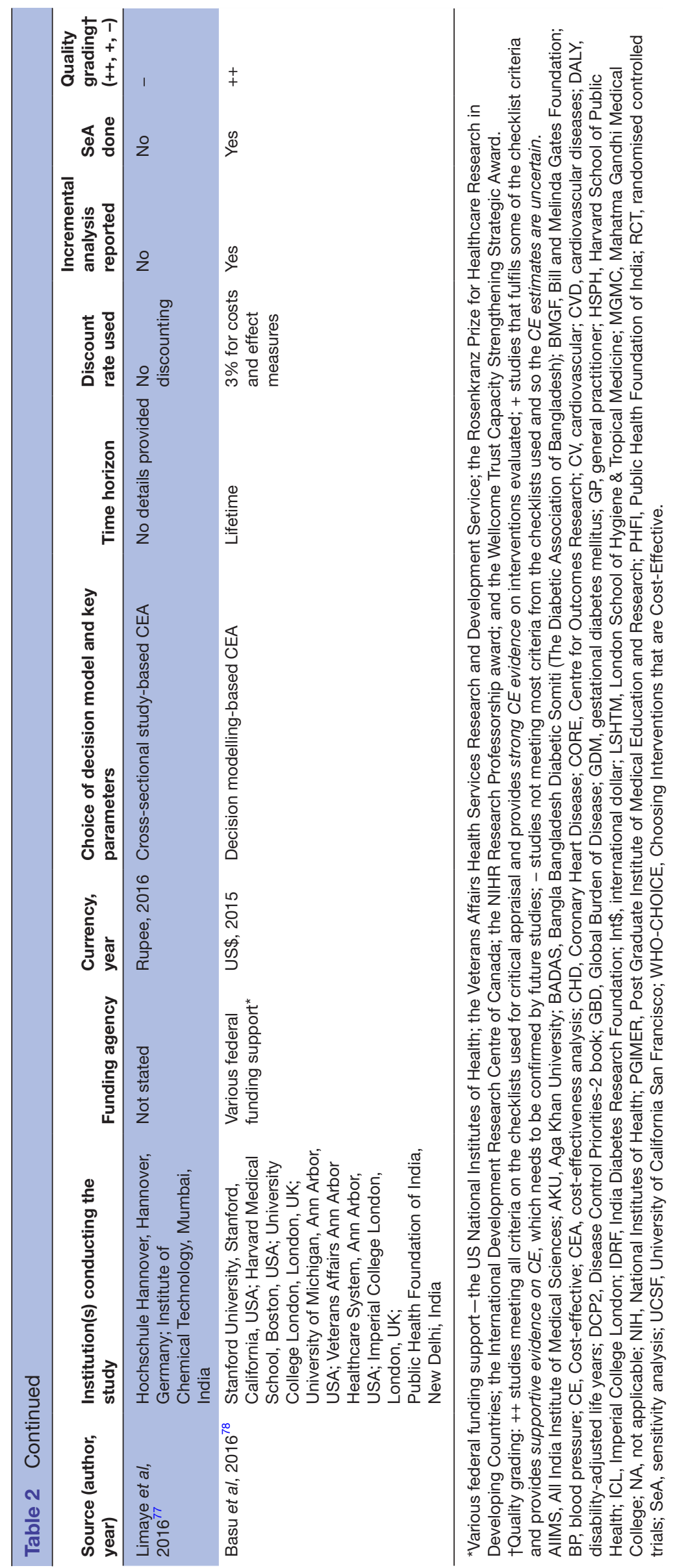


Table 3 Cost-effective interventions to control CVD and DM in South Asia

ICER, 2017†

Cost-effectiveness

threshold:

<GDP per capita per

Analytical Incremental Incremental effect QALY=green;

Intervention

Comparator

horizon capita (US\$)*

(DALY averted/

1-3×GDP per capita

per QALY=yellow

\section{Primordial prevention}

Policy interventions

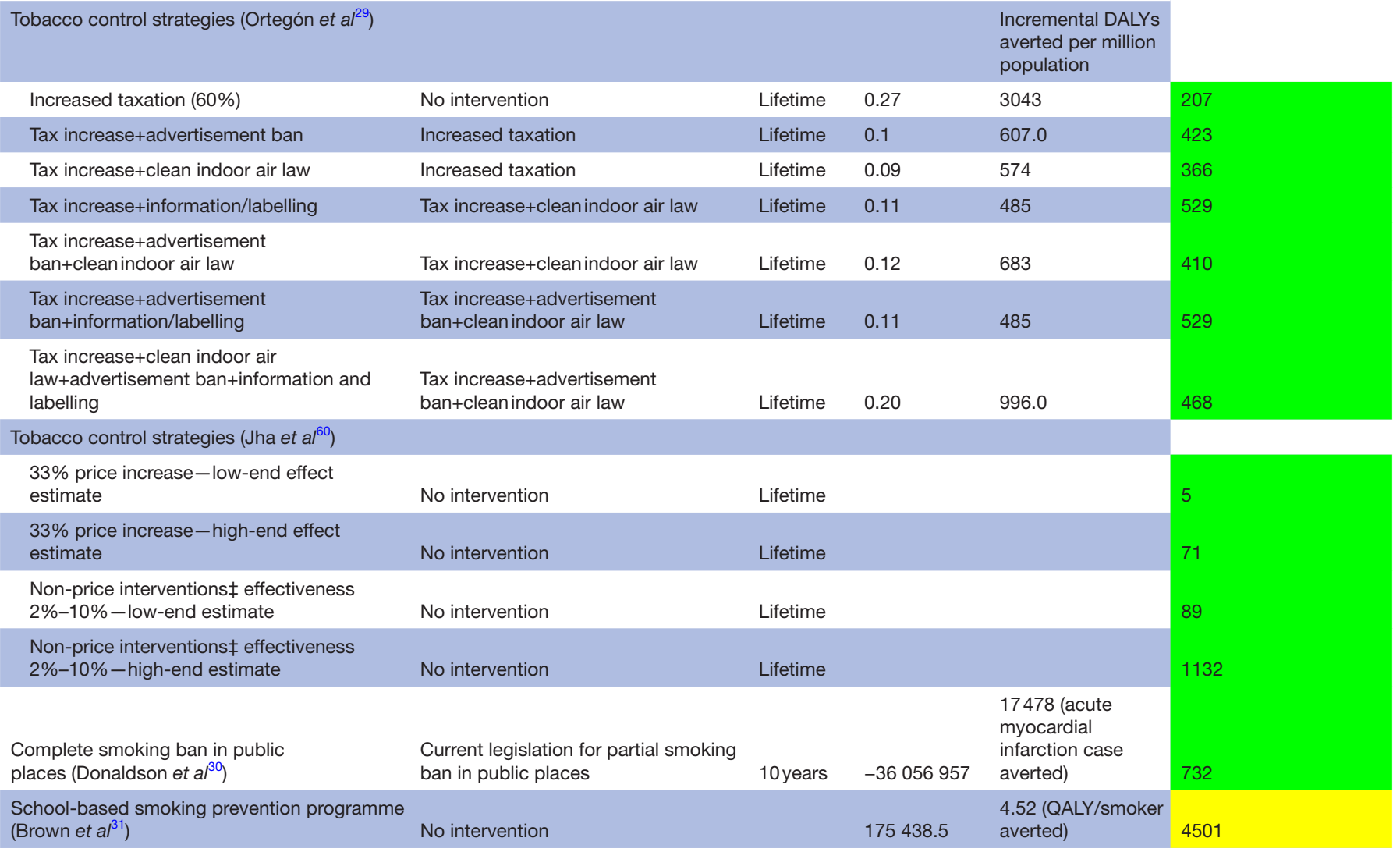

Promoting healthy diet strategies (Cecchini et $a^{\beta 2}$ )

\begin{tabular}{|c|c|c|c|}
\hline Food labelling & No intervention & 20 years & 2220 \\
\hline Fiscal measure for $100 \%$ population & No intervention & 50 years & Cost-saving \\
\hline Food advertising regulation & No intervention & 50 years & 774 \\
\hline \multicolumn{4}{|l|}{$\begin{array}{l}\text { Promoting healthy diet strategies (Murray et } \\
a^{57} \text { ) }\end{array}$} \\
\hline $\begin{array}{l}\text { Salt reduction through voluntary agreements } \\
\text { with industry }\end{array}$ & No intervention & Lifetime & 106 \\
\hline Health education through mass media & No intervention & Lifetime & 40 \\
\hline $\begin{array}{l}\text { Salt reduction via } \\
\text { legislation+health education via mass media }\end{array}$ & No intervention & Lifetime & 49 \\
\hline Promoting healthy diet strategies (Willett et $a l^{5}$ ) & & Lifetime & \\
\hline
\end{tabular}

Continued 
Table 3 Continued

\begin{tabular}{|c|c|c|c|c|c|}
\hline Intervention & Comparator & $\begin{array}{l}\text { Analytical } \\
\text { time } \\
\text { horizon }\end{array}$ & $\begin{array}{l}\text { Incremental } \\
\text { cost per } \\
\text { capita (US\$)* }\end{array}$ & $\begin{array}{l}\text { Incremental effect } \\
\text { (DALY averted/ } \\
\text { QALY gained)* }\end{array}$ & $\begin{array}{l}\text { ICER, 2017† } \\
\text { Cost-effectiveness } \\
\text { threshold: } \\
\text { <GDP per capita per } \\
\text { QALY=green; } \\
\text { 1-3×GDP per capita } \\
\text { per QALY=yellow }\end{array}$ \\
\hline $\begin{array}{l}\text { Substitute } 2 \% \text { of energy from trans fat with } \\
\text { polyunsaturated fatty acid ( } 7 \% \text { coronary } \\
\text { artery disease reduction at } \$ 0.6 \text { per adult) }\end{array}$ & No intervention & Lifetime & & & 2765 \\
\hline $\begin{array}{l}\text { Substitute } 2 \% \text { of energy from trans fat with } \\
\text { polyunsaturated fatty acid ( } 40 \% \text { coronary } \\
\text { artery disease reduction at } \$ 0.5 \text { per adult) }\end{array}$ & No intervention & Lifetime & & & Cost-saving \\
\hline $\begin{array}{l}\text { Substitute } 2 \% \text { of energy from trans fat with } \\
\text { polyunsaturated fatty acid ( } 40 \% \text { coronary } \\
\text { artery disease reduction at } \$ 0.6 \text { per adult) }\end{array}$ & No intervention & Lifetime & & & 376 \\
\hline $\begin{array}{l}\text { Reducing salt content by means of } \\
\text { legislation+public education }\end{array}$ & No intervention & Lifetime & & & 3613 \\
\hline $\begin{array}{l}\text { Blood pressure-lowering strategies (Rodgers } \\
\text { et } \mathrm{a} /^{59} \text { ) }\end{array}$ & & Lifetime & & & \\
\hline Prevention by salt legislation & No intervention & Lifetime & & & 49 \\
\hline \multicolumn{6}{|l|}{ Alcohol control strategies (Chisholm et $\mathrm{al}^{15}$ ) } \\
\hline Taxation current+25\% (alcohol use) & No intervention & Lifetime & & & Cost-saving \\
\hline Taxation current+50\% (alcohol use) & No intervention & Lifetime & & & Cost-saving \\
\hline Breath testing & No intervention & Lifetime & & & 152 \\
\hline Highest tax+advertisement ban & No intervention & Lifetime & & & 5002 \\
\hline \multicolumn{6}{|l|}{ Primary prevention } \\
\hline \multicolumn{6}{|l|}{ Policy interventions } \\
\hline $\begin{array}{l}\text { Universal screening for diabetes and } \\
\left.\text { hypertension (Dupka et } a l^{73}\right) \S\end{array}$ & & & & $\begin{array}{l}\text { DALY averted per } \\
\text { person }\end{array}$ & \\
\hline $\begin{array}{l}\text { Current Package of Essential Non- } \\
\text { Communicable (PEN) disease interventions } \\
\text { programme }\end{array}$ & No screening & Lifetime & -77.2 & 0.038 & Cost-saving \\
\hline Universal screening & Current WHO-PEN programme & Lifetime & -33.1 & 0.016 & Cost-saving \\
\hline $\begin{array}{l}\text { Screening for GDM to prevent DM (Lohse } \\
\text { et } a l^{66} \text { ) }\end{array}$ & No intervention & Lifetime & 26 & 2.33 & 16 \\
\hline Screening to prevent GDM (Marseille et $\left.a\right|^{35}$ ) & No intervention & Lifetime & 194358 & 120 & 2317 \\
\hline $\begin{array}{l}\text { Expansion of national insurance to cover } \\
\text { primary, secondary and tertiary treatment for } \\
\text { CVD (Basu et } a l^{39} \text { ) }\end{array}$ & & & & $\begin{array}{l}\text { Incremental DALY } \\
\text { averted per annum }\end{array}$ & \\
\hline $\begin{array}{l}\text { Insurance coverage for primary prevention } \\
\text { of CVD }\end{array}$ & Status quo & 20 years & 1.19 & 2544.5 & 528 \\
\hline \multicolumn{6}{|l|}{ Clinical interventions } \\
\hline \multicolumn{6}{|l|}{ Tobacco control strategies (Jha et $a l^{60}$ ) } \\
\hline $\begin{array}{l}\text { Nicotine replacement therapy effectiveness } \\
1 \%-5 \%-\text { low-end estimate }\end{array}$ & No intervention & Lifetime & & & 142 \\
\hline $\begin{array}{l}\text { Nicotine replacement therapy effectiveness } \\
1 \%-5 \%-\text { high-end estimate }\end{array}$ & No intervention & Lifetime & & & 1880 \\
\hline \multicolumn{6}{|l|}{ To reduce alcohol use (Chisholm et $\mathrm{al}^{15}$ ) } \\
\hline Brief physician advice & No intervention & Lifetime & & & 175 \\
\hline CVD prevention strategies (Ortegón et $a l^{29}$ ) & & & & $\begin{array}{l}\text { Incremental DALYs } \\
\text { averted per million } \\
\text { population }\end{array}$ & \\
\hline $\begin{array}{l}\text { Preventive multidrug treatment ( }>5 \% \text { risk } \\
\text { of CVD event) }\end{array}$ & No intervention & Lifetime & 1.97 & 4542 & 4238 \\
\hline $\begin{array}{l}\text { Preventive multidrug treatment ( }>35 \% \text { risk of } \\
\text { CVD event) }\end{array}$ & $\begin{array}{l}\text { Preventive multidrug treatment } \\
\text { ( }>5 \% \text { risk of CVD event) }\end{array}$ & Lifetime & 0.38 & 2582 & 341 \\
\hline $\begin{array}{l}\text { Combination of individual-based drug } \\
\text { therapy for hypertension and cholesterol } \\
\text { control }\end{array}$ & $\begin{array}{l}\text { Preventive multidrug treatment } \\
\text { ( }>5 \% \text { risk of CVD event) }\end{array}$ & Lifetime & 1.8 & 1780 & 2358 \\
\hline
\end{tabular}

Continued 
Table 3 Continued

ICER, 2017

Cost-effectiveness threshold:

$<$ GDP per capita per

Analytical Incremental Incremental effect QALY=green;

time cost per (DALY averted/1-3×GDP per capita

Intervention

Comparator

horizon capita (US\$)*

ALY averted/

per QALY=yellow

Combined home health education plus

trained general practitioner for hypertension

management (Jafar et $a l^{36}$ )

No intervention

2 years

48

Diabetes prevention strategies (Narayan

et $\mathrm{al}^{34}$ )

\begin{tabular}{|c|c|c|c|c|}
\hline $\begin{array}{l}\text { Smoking cessation (physician counselling } \\
\text { and nicotine replacement therapy) }\end{array}$ & No intervention & Lifetime & & \\
\hline $\begin{array}{l}\text { Preconception care for women of } \\
\text { reproductive age }\end{array}$ & No intervention & Lifetime & & \\
\hline $\begin{array}{l}\text { Lifestyle interventions to prevent type } 2 \\
\text { diabetes }\end{array}$ & No intervention & Lifetime & & \\
\hline $\begin{array}{l}\text { Metformin intervention to prevent type } 2 \\
\text { diabetes }\end{array}$ & No intervention & Lifetime & & \\
\hline $\begin{array}{l}\text { Lifestyle modification+metformin to prevent } \\
\text { type } 2 \text { diabetes (Ramachandran et } a^{37} \text { ) }\end{array}$ & & & & $\begin{array}{l}\text { Number needed to } \\
\text { treat to prevent a } \\
\text { case of diabetes }\end{array}$ \\
\hline Lifestyle modification & Standard healthcare advice & 3 years & 164 & 6.4 \\
\hline Metformin & Standard healthcare advice & 3 years & 159 & 6.9 \\
\hline Lifestyle modification+metformin & Standard healthcare advice & 3 years & 209 & 6.5 \\
\hline
\end{tabular}

1990.6

Cost-saving

163.6

4962.9

2302

2396

2973

Policy interventions

Policies to expand use of drugs for acute myocardial infarction (Megiddo et $\mathrm{al}^{38}$ )

Acute myocardial infarction treatment

\begin{tabular}{|c|c|c|c|c|c|}
\hline Aspirin to baseline & No intervention & Lifetime & & & 0.6 \\
\hline Aspirin+injection streptokinase & Aspirin to baseline & Lifetime & & & 693 \\
\hline \multicolumn{6}{|l|}{ Acute myocardial infarction prevention } \\
\hline Aspirin to baseline & No intervention & Lifetime & & & 299 \\
\hline Aspirin+BB & Aspirin to baseline & Lifetime & & & 1960 \\
\hline Aspirin+BB+ACEi & Aspirin+BB & Lifetime & & & 3120 \\
\hline Polypill to baseline & Aspirin+BB+ACEi+statin & Lifetime & & & 1904 \\
\hline $\begin{array}{l}\text { Expansion of national insurance to cover } \\
\text { primary, secondary and tertiary treatment for } \\
\text { CVD (Basu et al) })^{39}\end{array}$ & & & & $\begin{array}{l}\text { Incremental DALY } \\
\text { averted per annum }\end{array}$ & \\
\hline $\begin{array}{l}\text { Insurance coverage for secondary } \\
\text { prevention of CVD }\end{array}$ & Status quo & 20 years & 0.36 & 147.9 & 2708 \\
\hline $\begin{array}{l}\text { Insurance coverage for tertiary treatment } \\
\text { of CVD }\end{array}$ & Status quo & 20 years & 4.68 & 2076.8 & 2538 \\
\hline \multicolumn{6}{|l|}{ Clinical interventions } \\
\hline CVD treatment strategies (Ortegón et $a l^{29}$ ) & & & & $\begin{array}{l}\text { Incremental DALYs } \\
\text { averted per million } \\
\text { population }\end{array}$ & \\
\hline Treatment of CHF with diuretics & No intervention & Lifetime & 0.03 & 402 & 188.9 \\
\hline $\begin{array}{l}\text { Treatment of } \mathrm{CHF} \text { with diuretics+exercise } \\
\text { training }\end{array}$ & Treatment of $\mathrm{CHF}$ with diuretics & Lifetime & 0.02 & 60 & 776.6 \\
\hline $\begin{array}{l}\text { Treatment of } \mathrm{CHF} \text { with } \\
\text { diuretics+exercise training+ACEi }\end{array}$ & Treatment of $\mathrm{CHF}$ with diuretics & Lifetime & 0.04 & 72 & 1296.7 \\
\hline $\begin{array}{l}\text { Treatment of } \mathrm{CHF} \text { with } \\
\text { diuretics+exercise training+BB }\end{array}$ & Treatment of $\mathrm{CHF}$ with diuretics & Lifetime & 0.08 & 95 & 1963 \\
\hline $\begin{array}{l}\text { Treatment of post-acute ischaemic heart } \\
\text { disease and stroke with aspirin, BB, statin }\end{array}$ & No intervention & Lifetime & 0.03 & 609 & 114 \\
\hline
\end{tabular}

Continued 
Table 3 Continued

\begin{tabular}{|c|c|c|c|c|c|}
\hline Intervention & Comparator & $\begin{array}{l}\text { Analytical } \\
\text { time } \\
\text { horizon }\end{array}$ & $\begin{array}{l}\text { Incremental } \\
\text { cost per } \\
\text { capita (US\$)* }\end{array}$ & $\begin{array}{l}\text { Incremental effect } \\
\text { (DALY averted/ } \\
\text { QALY gained)* }\end{array}$ & $\begin{array}{l}\text { <GDP per capita per } \\
\text { QALY=green; } \\
\text { 1-3×GDP per capita } \\
\text { per QALY=yellow }\end{array}$ \\
\hline $\begin{array}{l}\text { Treatment of acute myocardial infarction } \\
\text { (aspirin, BB, ACEi, streptokinase)+post- } \\
\text { acute ischaemic heart disease with aspirin, } \\
\text { BB, statin, ACEi }\end{array}$ & $\begin{array}{l}\text { Treatment of acute myocardial } \\
\text { infarction (aspirin, BB, ACEi, } \\
\text { streptokinase)+post-acute ischaemic } \\
\text { heart disease with aspirin, BB, statin }\end{array}$ & Lifetime & 0.37 & 945 & 914 \\
\hline $\begin{array}{l}\text { Treatment of acute myocardial infarction } \\
\text { (aspirin, BB, ACEi, streptokinase)+post- } \\
\text { acute ischaemic heart disease and stroke } \\
\text { with aspirin, BB, statin }\end{array}$ & No intervention & Lifetime & 0.04 & 263 & 354 \\
\hline $\begin{array}{l}\text { Treatment of acute myocardial infarction } \\
\text { (aspirin, BB, ACEi, streptokinase)+post- } \\
\text { acute ischaemic heart disease and stroke } \\
\text { with aspirin, BB, statin+CHF (diuretic, } \\
\text { exercise) }\end{array}$ & $\begin{array}{l}\text { Treatment of acute myocardial } \\
\text { infarction (aspirin, BB, ACEi, } \\
\text { streptokinase)+post-acute ischaemic } \\
\text { heart disease and stroke with aspirin, } \\
\text { BB, statin }\end{array}$ & Lifetime & 0.26 & 1879 & 321 \\
\hline $\begin{array}{l}\text { Individual-based prevention (hypertension } \\
\text { and cholesterol control)+treatment of acute } \\
\text { myocardial infarction (aspirin, BB, ACEi, } \\
\text { streptokinase)+post-acute ischaemic heart } \\
\text { disease (aspirin, BB, ACEi, statin) }\end{array}$ & $\begin{array}{l}\text { Individual-based prevention } \\
\text { (hypertension and cholesterol } \\
\text { control)+treatment of acute myocardial } \\
\text { infarction (aspirin, BB, ACEi, } \\
\text { streptokinase)+post-acute ischaemic } \\
\text { heart disease (aspirin, BB, statin) }\end{array}$ & Lifetime & 0.04 & 250 & 373 \\
\hline $\begin{array}{l}\text { Individual-based prevention (hypertension } \\
\text { and cholesterol control)+treatment of acute } \\
\text { myocardial infarction (aspirin, BB, ACEi, } \\
\text { streptokinase)+post-acute ischaemic heart } \\
\text { disease and stroke (aspirin, BB, statin) }\end{array}$ & $\begin{array}{l}\text { Individual-based prevention } \\
\text { (hypertension and cholesterol } \\
\text { control)+treatment of acute myocardial } \\
\text { infarction (aspirin, BB, ACEi, } \\
\text { streptokinase)+post-acute ischaemic } \\
\text { heart disease (aspirin, BB, ACEi, } \\
\text { statin) }\end{array}$ & Lifetime & 0.04 & 201 & 464 \\
\hline $\begin{array}{l}\text { Individual-based prevention (hypertension } \\
\text { and cholesterol control)+treatment of acute } \\
\text { myocardial infarction (aspirin, BB, ACEi, } \\
\text { streptokinase)+post-acute ischaemic heart } \\
\text { disease and stroke (aspirin, BB, statin)+CHF } \\
\text { (diuretic, exercise) }\end{array}$ & $\begin{array}{l}\text { Individual-based prevention } \\
\text { (hypertension and cholesterol } \\
\text { control)+treatment of post-acute } \\
\text { ischaemic heart disease and stroke } \\
\text { (aspirin, BB, statin)+CHF (diuretic, } \\
\text { exercise) }\end{array}$ & Lifetime & 0.26 & 437 & 1387 \\
\hline $\begin{array}{l}\text { Combination drug treatment (>25\% risk } \\
\text { of CVD event)+treatment of acute } \\
\text { myocardial infarction (aspirin, BB, ACEi, } \\
\text { streptokinase)+treatment of post-acute } \\
\text { ischaemic heart disease (aspirin, BB, statin) }\end{array}$ & No intervention & Lifetime & 1.16 & 4852 & 557 \\
\hline $\begin{array}{l}\text { Combination drug treatment (>25\% risk } \\
\text { of CVD event)+treatment of acute } \\
\text { myocardial infarction (aspirin, BB, ACEi, } \\
\text { streptokinase)+treatment of post-acute } \\
\text { ischaemic heart disease (aspirin, BB, ACEi, } \\
\text { statin) }\end{array}$ & $\begin{array}{l}\text { Combination drug treatment } \\
\text { ( }>25 \% \text { risk of CVD event)+treatment of } \\
\text { acute myocardial infarction (aspirin, } \\
\text { BB, ACEi, streptokinase)+treatment of } \\
\text { post-acute ischaemic heart disease } \\
\text { (aspirin, BB, statin) }\end{array}$ & Lifetime & 0.04 & 237 & 394 \\
\hline $\begin{array}{l}\text { Combination drug treatment ( }>25 \% \text { risk } \\
\text { of CVD event)+treatment of acute } \\
\text { myocardial infarction (aspirin, BB, ACEi, } \\
\text { streptokinase)+treatment of post-acute } \\
\text { ischaemic heart disease and stroke (aspirin, } \\
\text { BB, statin) }\end{array}$ & $\begin{array}{l}\text { Combination drug treatment } \\
\text { ( }>25 \% \text { risk of CVD event)+treatment of } \\
\text { acute myocardial infarction (aspirin, } \\
\text { BB, ACEi, streptokinase)+treatment of } \\
\text { post-acute ischaemic heart disease } \\
\text { (aspirin, BB, ACEi, statin) }\end{array}$ & Lifetime & 0.04 & 178 & 524 \\
\hline
\end{tabular}


Table 3 Continued

\begin{tabular}{|c|c|c|c|c|c|}
\hline Intervention & Comparator & $\begin{array}{l}\text { Analytical } \\
\text { time } \\
\text { horizon }\end{array}$ & $\begin{array}{l}\text { Incremental } \\
\text { cost per } \\
\text { capita (US\$)* }\end{array}$ & $\begin{array}{l}\text { Incremental effect } \\
\text { (DALY averted/ } \\
\text { QALY gained)* }\end{array}$ & $\begin{array}{l}\text { QALY=green; } \\
\text { 1-3×GDP per capita } \\
\text { per QALY=yellow }\end{array}$ \\
\hline $\begin{array}{l}\text { Combination drug treatment ( }>25 \% \text { risk } \\
\text { of CVD event)+treatment of post-acute } \\
\text { ischaemic heart disease and stroke (aspirin, } \\
\mathrm{BB} \text {, statin)+CHF (diuretics, exercise) }\end{array}$ & $\begin{array}{l}\text { Combination drug treatment } \\
\text { (>25\% risk of CVD event)+treatment of } \\
\text { acute myocardial infarction (aspirin, } \\
\mathrm{BB}, \mathrm{ACEi} \text {, streptokinase)+treatment of } \\
\text { post-acute ischaemic heart disease } \\
\text { and stroke (aspirin, BB, statin) }\end{array}$ & Lifetime & -0.23 & 32 & Cost-saving \\
\hline $\begin{array}{l}\text { Preventive multidrug treatment for }>25 \% \text { risk } \\
\text { of CVD event+multidrug treatment of } \\
\text { acute myocardial infarction or post- } \\
\text { acute ischaemic heart disease and } \\
\text { stroke+diuretics and exercise for CHF }\end{array}$ & $\begin{array}{l}\text { Combination drug treatment } \\
\text { ( }>25 \% \text { risk of CVD event)+treatment of } \\
\text { post-acute ischaemic heart disease } \\
\text { and stroke (aspirin, BB, statin)+CHF } \\
\text { (diuretics, exercise) }\end{array}$ & Lifetime & 0.26 & 558 & 1086 \\
\hline $\begin{array}{l}\text { Combination drug treatment ( }>35 \% \text { risk } \\
\text { of CVD event)+treatment of post-acute } \\
\text { ischaemic heart disease and stroke (aspirin, } \\
\text { BB, statin)+CHF (diuretics, exercise) }\end{array}$ & $\begin{array}{l}\text { Combination drug treatment } \\
\text { (>35\% risk of CVD event)+treatment of } \\
\text { post-acute ischaemic heart disease } \\
\text { and stroke (aspirin, BB, statin)+CHF } \\
\text { (diuretics, exercise) }\end{array}$ & Lifetime & -0.23 & 31 & Cost-saving \\
\hline $\begin{array}{l}\text { Preventive multidrug treatment for }>35 \% \text { risk } \\
\text { of CVD event+multidrug treatment of } \\
\text { acute myocardial infarction or post- } \\
\text { acute ischaemic heart disease and } \\
\text { stroke+diuretics and exercise for CHF }\end{array}$ & $\begin{array}{l}\text { Combination drug treatment } \\
\text { (>35\% risk of CVD event)+treatment of } \\
\text { post-acute ischaemic heart disease } \\
\text { and stroke (aspirin, BB, statin)+CHF } \\
\text { (diuretics, exercise) }\end{array}$ & Lifetime & 0.26 & 630 & 963 \\
\hline \multicolumn{6}{|l|}{ CVD treatment strategies (Murray et $a l^{57}$ ) } \\
\hline $\begin{array}{l}\text { Treatment of SBP above } 160 \mathrm{~mm} \mathrm{Hg} \text { with BB } \\
\text { and diuretic }\end{array}$ & No intervention & Lifetime & & & 103.2 \\
\hline $\begin{array}{l}\text { Treatment of SBP above } 140 \mathrm{~mm} \mathrm{Hg} \text { with BB } \\
\text { and diuretic }\end{array}$ & No intervention & Lifetime & & & 257.9 \\
\hline $\begin{array}{l}\text { Treatment with statins for total cholesterol } \\
\text { concentrations above education } 6.2 \mathrm{mmol} / \mathrm{L}\end{array}$ & No intervention & Lifetime & & & 134.7 \\
\hline $\begin{array}{l}\text { Treatment with statins for total cholesterol } \\
\text { concentrations above education } 5.7 \mathrm{mmol} / \mathrm{L}\end{array}$ & No intervention & Lifetime & & & 203.5 \\
\hline $\begin{array}{l}\text { Treatment of SBP above } 140 \mathrm{~mm} \mathrm{Hg} \text { with } \\
\text { BB and diuretics and with statins for total } \\
\text { cholesterol concentrations above } 6.2 \mathrm{mmol} / \mathrm{L}\end{array}$ & No intervention & Lifetime & & & 240.7 \\
\hline $\begin{array}{l}\text { Multiple drug therapy in }>35 \% \text { CV risk over } \\
10 \text { years }\end{array}$ & No intervention & Lifetime & & & Cost-saving \\
\hline $\begin{array}{l}\text { Multiple drug therapy in }>25 \% \text { CV risk over } \\
10 \text { years }\end{array}$ & No intervention & Lifetime & & & 94.6 \\
\hline $\begin{array}{l}\text { Multiple drug therapy in }>15 \% \text { CV risk over } \\
10 \text { years }\end{array}$ & No intervention & Lifetime & & & 137.5 \\
\hline $\begin{array}{l}\text { Multiple drug therapy in }>5 \% \text { CV risk over } \\
10 \text { years }\end{array}$ & No intervention & Lifetime & & & 220.7 \\
\hline
\end{tabular}

CVD treatment and secondary prevention

(Gaziano et $\mathrm{a}^{43}$ )

\begin{tabular}{lll}
$\begin{array}{l}\text { Medical therapy for acute myocardial } \\
\text { infarction with aspirin }\end{array}$ & No intervention & Lifetime \\
$\begin{array}{l}\text { Medical therapy for acute myocardial } \\
\text { infarction with aspirin+BB }\end{array}$ & No intervention & Lifetime \\
$\begin{array}{l}\text { Medical therapy for acute myocardial } \\
\text { infarction with aspirin+BB+streptokinase }\end{array}$ & No intervention & Lifetime \\
$\begin{array}{l}\text { Medical therapy (aspirin+BB) for ischaemic } \\
\text { heart disease, having hospital access }\end{array}$ & No intervention & Lifetime \\
$\begin{array}{l}\text { Medical therapy (aspirin+BB+ACEi) for } \\
\text { ischaemic heart disease, having hospital } \\
\text { access }\end{array}$ & No intervention & Lifetime \\
$\begin{array}{l}\text { Medical therapy (aspirin+BB+ACEi+statin) } \\
\text { for ischaemic heart disease, having hospital } \\
\text { access }\end{array}$ & No intervention & \\
$\begin{array}{l}\text { Medical therapy (aspirin+BB) for ischaemic } \\
\text { heart disease, limited hospital access }\end{array}$ & No intervention & Lifetime \\
\hline
\end{tabular}

Continued 


\begin{tabular}{|c|c|c|c|c|c|}
\hline Intervention & Comparator & $\begin{array}{l}\text { Analytical } \\
\text { time } \\
\text { horizon }\end{array}$ & $\begin{array}{l}\text { Incremental } \\
\text { cost per } \\
\text { capita (US\$)* }\end{array}$ & $\begin{array}{l}\text { Incremental effect } \\
\text { (DALY averted/ } \\
\text { QALY gained)* }\end{array}$ & $\begin{array}{l}\text { ICER, 2017† } \\
\text { Cost-effectiveness } \\
\text { threshold: } \\
\text { <GDP per capita per } \\
\text { QALY=green; } \\
\text { 1-3×GDP per capita } \\
\text { per QALY=yellow }\end{array}$ \\
\hline $\begin{array}{l}\text { Medical therapy (aspirin+BB+ACEi) for } \\
\text { ischaemic heart disease, limited hospital } \\
\text { access }\end{array}$ & No intervention & Lifetime & & & 2373.4 \\
\hline ACEi for CHF, hospital access & Baseline of diuretics & Lifetime & & & Cost-saving \\
\hline $\begin{array}{l}\text { ACEi, BB (metoprolol) for CHF, hospital } \\
\text { access }\end{array}$ & Baseline of diuretics & Lifetime & & & 627.7 \\
\hline ACEi for CHF, limited hospital access & Baseline of diuretics & Lifetime & & & 71.6 \\
\hline $\begin{array}{l}\text { ACEi, BB (metoprolol) for CHF, limited } \\
\text { hospital access }\end{array}$ & Baseline of diuretics & Lifetime & & & 782.5 \\
\hline \multicolumn{6}{|l|}{$\begin{array}{l}\text { Blood pressure-lowering strategies (Rodgers } \\
\text { et } a^{59} \text { ) }\end{array}$} \\
\hline $\begin{array}{l}\text { Multidrug regimen (aspirin, a BB, a thiazide } \\
\text { diuretic, an } \mathrm{ACEi} \text { and a statin) in } 35 \% \mathrm{CV} \\
\text { risk over } 10 \text { years }\end{array}$ & No intervention & Lifetime & & & 1827 \\
\hline $\begin{array}{l}\text { Multidrug regimen (aspirin, a BB, a thiazide } \\
\text { diuretic, an ACEi and a statin) in } 25 \% \mathrm{CV} \\
\text { risk over } 10 \text { years }\end{array}$ & No intervention & Lifetime & & & 3408.6 \\
\hline $\begin{array}{l}\text { Multidrug regimen (aspirin, a BB, a thiazide } \\
\text { diuretic, an ACEi and a statin) in } 15 \% \mathrm{CV} \\
\text { risk over } 10 \text { years }\end{array}$ & No intervention & Lifetime & & & 5268.2 \\
\hline \multicolumn{6}{|l|}{$\begin{array}{l}\text { Treat-to-target, benefit-based tailored } \\
\text { treatment strategy vs hybrid strategy for } \\
\text { lowering CVD risk (Basu et } a l^{78} \text { ) }\end{array}$} \\
\hline $\begin{array}{l}\text { People treated identically by all three } \\
\text { strategies }\end{array}$ & No intervention & 10 years & & & 383.7 \\
\hline $\begin{array}{l}\text { People treated most intensively by treat-to- } \\
\text { target }\end{array}$ & No intervention & 10 years & & & 432.1 \\
\hline $\begin{array}{l}\text { People treated most intensively by benefit- } \\
\text { based tailored treatment }\end{array}$ & No intervention & 10 years & & & 206.1 \\
\hline People treated most intensively by hybrid & No intervention & 10 years & & & 384.4 \\
\hline $\begin{array}{l}\text { Prehospital ECG for accurate referral and } \\
\text { timely access to reperfusion (Schulman- } \\
\text { Marcus et } a l^{40} \text { ) }\end{array}$ & $\begin{array}{l}\text { No ECG-based referral in case of } \\
\text { chest pain }\end{array}$ & Lifetime & 0.15 & $\begin{array}{l}0.012(\mathrm{QALY} \\
\text { gained) }\end{array}$ & 26.1 \\
\hline $\begin{array}{l}\text { Diabetes treatment strategies (Narayan } \\
\text { et } \mathrm{a}^{34} \text { ) }\end{array}$ & & Lifetime & & & \\
\hline $\begin{array}{l}\text { Glycaemic control in people with } \\
\mathrm{HbA} 1 \mathrm{c}>9 \% \text { (insulin, oral glucose-lowering } \\
\text { agents, diet and exercise) }\end{array}$ & No intervention & Lifetime & & & Cost-saving \\
\hline $\begin{array}{l}\text { Blood pressure control in people with } \\
>160 / 95 \mathrm{~mm} \mathrm{Hg}\end{array}$ & No intervention & Lifetime & & & Cost-saving \\
\hline Foot care in people with a high risk of ulcers & No intervention & Lifetime & & & Cost-saving \\
\hline Influenza vaccination among elderly & No intervention & Lifetime & & & 490.8 \\
\hline Annual eye examination & No intervention & Lifetime & & & 954.4 \\
\hline ACEi use for people with diabetes & No intervention & Lifetime & & & 1390.7 \\
\hline $\begin{array}{l}\text { Intensive glucose control for people with } \\
\text { HbA1c }>8 \% \text { (insulin, oral glucose-lowering } \\
\text { agents or both) }\end{array}$ & No intervention & Lifetime & & & 5453.7 \\
\hline $\begin{array}{l}\text { Treatment of diabetes and its } \\
\text { complications (Ortegón et }\left.a\right|^{29} \text { ) }\end{array}$ & & & & $\begin{array}{l}\text { Incremental DALYs } \\
\text { averted per million } \\
\text { population }\end{array}$ & \\
\hline Standard glycaemic control & No intervention & Lifetime & 0.82 & 1717 & 1115 \\
\hline $\begin{array}{l}\text { Retinopathy screening and photocoagulation } \\
\text { therapy }\end{array}$ & No intervention & Lifetime & 0.32 & 1891 & 396.4 \\
\hline $\begin{array}{l}\text { Standard glycaemic control+retinopathy } \\
\text { screening+neuropathy screening }\end{array}$ & $\begin{array}{l}\text { Intensive glycaemic } \\
\text { control+neuropathy screening }\end{array}$ & Lifetime & -0.65 & 213 & Cost-saving \\
\hline
\end{tabular}


Table 3 Continued

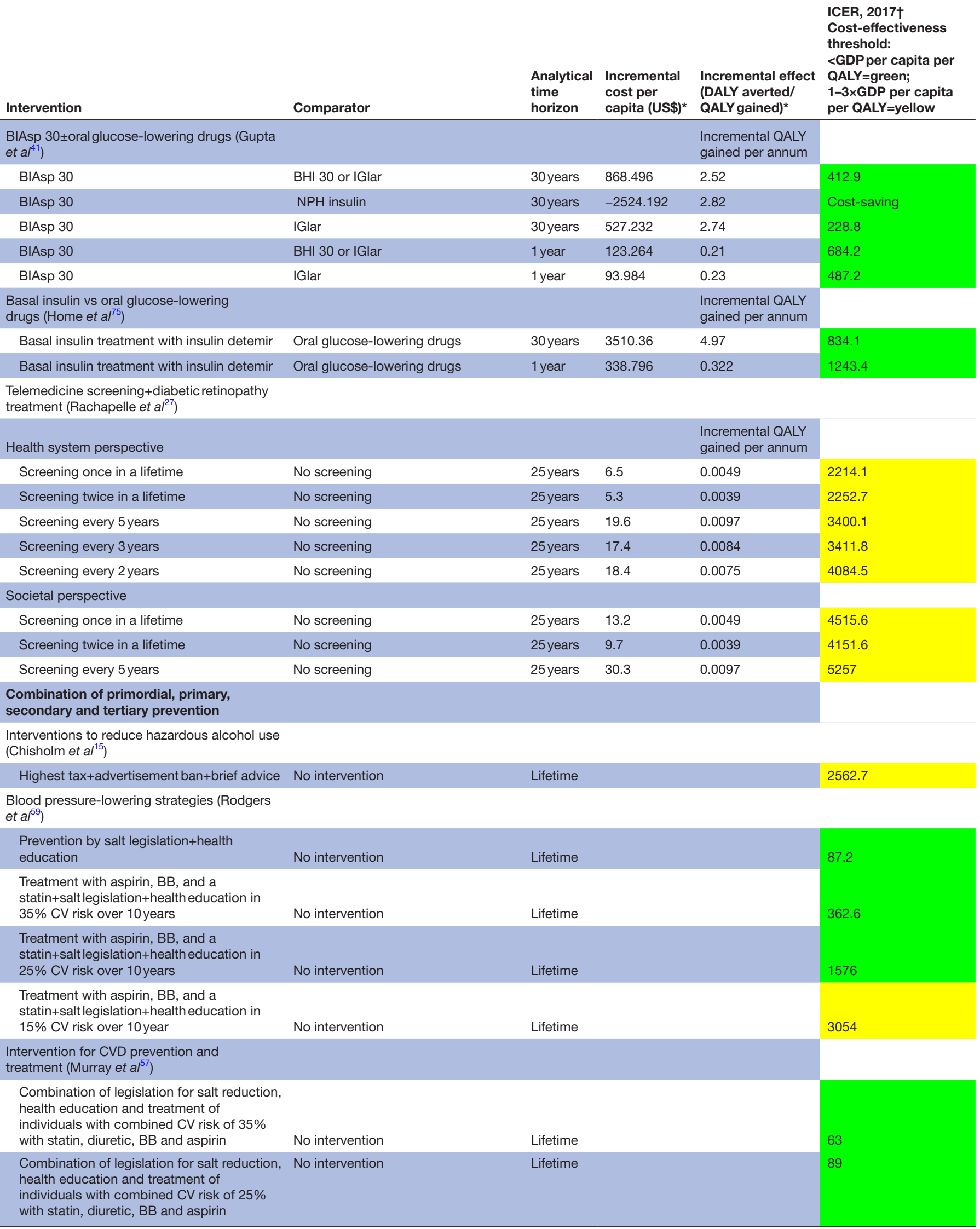

Continued 


\begin{tabular}{|c|c|c|c|c|c|}
\hline Intervention & Comparator & $\begin{array}{l}\text { Analytical } \\
\text { time } \\
\text { horizon }\end{array}$ & $\begin{array}{l}\text { Incremental } \\
\text { cost per } \\
\text { capita (US\$)* }\end{array}$ & $\begin{array}{l}\text { Incremental effect } \\
\text { (DALY averted/ } \\
\text { QALY gained)* }\end{array}$ & $\begin{array}{l}\text { <GDP per capita per } \\
\text { QALY=green; } \\
\text { 1-3×GDP per capita } \\
\text { per QALY=yellow }\end{array}$ \\
\hline $\begin{array}{l}\text { Combination of legislation for salt reduction, } \\
\text { health education and treatment of } \\
\text { individuals with combined CV risk of } 15 \% \\
\text { with statin, diuretic, BB and aspirin }\end{array}$ & No intervention & Lifetime & & & 132 \\
\hline $\begin{array}{l}\text { Combination of legislation for salt } \\
\text { reduction, health education and treatment } \\
\text { of individuals with combined CV risk of } 5 \% \\
\text { with statin, diuretic, BB and aspirin }\end{array}$ & No intervention & Lifetime & & & 212 \\
\hline
\end{tabular}

CVD prevention and treatment

strategies (Ortegón et $a 2^{29}$ )

Population-based prevention (hypertension and cholesterol control)+treatment of acute myocardial infarction (aspirin, BB, ACEi, streptokinase)+post-acute ischaemic heart disease (aspirin, BB, statin)

Population-based prevention (hypertension and cholesterol control)+treatment of acute myocardial infarction (aspirin, BB, ACEi, streptokinase)+post-acute ischaemic heart disease (aspirin, BB, ACEi, statin)

\begin{tabular}{|c|c|c|c|c|c|}
\hline disease (aspirin, BB, ACEi, statin) & heart disease (aspirin, BB, statin) & Lifetime & 0.04 & 285 & 326 \\
\hline $\begin{array}{l}\text { Population-based prevention (hypertension } \\
\text { and cholesterol control)+treatment of acute } \\
\text { myocardial infarction (aspirin, BB, ACEi, } \\
\text { streptokinase)+post-acute ischaemic heart } \\
\text { disease and stroke (aspirin, BB, statin) }\end{array}$ & $\begin{array}{l}\text { Population-based prevention } \\
\text { (hypertension and cholesterol } \\
\text { control)+treatment of acute myocardial } \\
\text { infarction (aspirin, BB, ACEi, } \\
\text { streptokinase)+post-acute ischaemic } \\
\text { heart disease (aspirin, BB, ACEi, } \\
\text { statin) }\end{array}$ & Lifetime & 0.04 & 246 & 380 \\
\hline $\begin{array}{l}\text { Population-based prevention (hypertension } \\
\text { and cholesterol control)+treatment of acute } \\
\text { myocardial infarction (aspirin, BB, ACEi, } \\
\text { streptokinase)+post-acute Ischaemic heart } \\
\text { disease and stroke (aspirin, BB, statin)+CHF } \\
\text { (diuretic, exercise) }\end{array}$ & $\begin{array}{l}\text { Population-based prevention } \\
\text { (hypertension and cholesterol } \\
\text { control)+treatment of post-acute } \\
\text { ischaemic heart disease and stroke } \\
\text { (aspirin, BB, statin)+CHF (diuretic, } \\
\text { exercise) }\end{array}$ & Lifetime & 0.26 & 646 & 937 \\
\hline $\begin{array}{l}\text { Expansion of national insurance to cover } \\
\text { primary, secondary and tertiary treatment for } \\
\text { CVD (Basu et al) }\end{array}$ & & & & $\begin{array}{l}\text { Incremental DALY } \\
\text { averted per annum }\end{array}$ & \\
\hline $\begin{array}{l}\text { Insurance coverage for } \\
\text { primary+secondary prevention of CVD }\end{array}$ & Primary prevention only & 20 years & 0.35 & 145.0 & 2739 \\
\hline $\begin{array}{l}\text { Insurance coverage for } \\
\text { primary+tertiary prevention of CVD }\end{array}$ & Primary prevention only & 20 years & 4.67 & 2084.6 & 2525 \\
\hline
\end{tabular}

GDP per capita (US\$, 2016) for India, Pakistan and Bhutan are 1861.5, 1468.2 and 729.5, respectively.

*Values refer to original study period.

†Conversion to current year, based on midyear consumer price index inflation rates.

$\ddagger$ Non-price interventions to reduce tobacco use:

-protection from exposure to tobacco smoke

-regulation of the contents of tobacco products

-regulation of tobacco product disclosures

-packaging and labelling of tobacco products

-education, communication, training and public awareness

-tobacco advertising, promotion and sponsorship

-demand reduction measures concerning tobacco dependence and cessation.

§Conducted in Bhutan.

TConducted in Pakistan.

ACEi, ACE inhibitors; BB, beta-blockers (blood pressure-lowering agents; BHI, biphasic human insulin; BIAsp 30, biphasic insulin aspart 30; CHF, congestive heart failure; CV, cardiovascular; CVD, cardiovascular diseases; DALY, disability-adjusted life years; DM, diabetes mellitus; GDM, gestation diabetes mellitus; GDP, gross domestic product; HbA1c, glycated haemoglobin; ICER, incremental cost-effectiveness ratio; IGlar, insulin glargine; QALY, quality-adjusted life years; NPH, neutral protamine Hagedorn; SBP, systolic blood pressure.

hypertension management was highly cost-effective per DALY averted than individual strategies or no intervention in Pakistan. ${ }^{36}$
Lifestyle modification (weight reduction, increased activity and healthy diet) was most cost-effective for prevention of DM, followed by metformin alone and 
combination of lifestyle modification plus metformin (1-3×GDP per capita) ${ }^{37}$

\section{Secondary and tertiary prevention}

Policies to expand access of drugs for acute myocardial infarction prevention and treatment were cost-effective per DALY averted. ${ }^{38}$ Also, expansion of national insurance to cover secondary or tertiary prevention of CVD was most cost-effective per QALY gained compared with status quo. ${ }^{39}$ Clinical interventions for secondary prevention of CVD are mostly cost-effective per DALY averted. ${ }^{29}$ ECG-based doctor referral to cardiac care unit versus no 'ECG use' was cost-effective per QALY gained. ${ }^{40}$

Many strategies for DM treatment and secondary prevention of macrovascular and microvascular complications were found to be highly cost-effective or cost-effective. Examples of highly cost-effective interventions are glycaemic control in people with glycated haemoglobin (A1c) $>9 \%$ with insulin, oral glucose-lowering drugs, diet and exercise, BP control in people with $>165 / 95 \mathrm{~mm}$ $\mathrm{Hg}$, and foot care in people with high risk of ulcers $(<1 \times$ GDP per capita per DALY averted $) .{ }^{34}$ Basal insulin treatment versus oral glucose-lowering drugs was highly cost-effective $(<1 \times$ GDP per capita per QALY gained $){ }^{41}$ Diabetic retinopathy screening every $2-5$ years versus no screening was cost-effective $(1-3 \times$ GDP per capita per QALY gained). ${ }^{27}$

\section{Combination of primordial, primary, secondary and tertiary prevention}

Multicomponent strategies of salt reduction through legislation (increase tax), health education, plus treatment of individuals at 35\% cardiovascular risk with statin, diuretic, beta-blockers and aspirin were highly cost-effective, followed by similar strategy in those at $25 \%$ or $15 \%$ cardiovascular risk over 10 years. ${ }^{29}$ Policy measures such as expansion of insurance coverage for primary, secondary and tertiary prevention of CVD were also cost-effective (1-3×GDP per capita per DALY averted) ${ }^{39}$

Interventions that resulted in ICER $>3 \times$ GDP per capita or were dominated by other highly cost-effective strategies are presented in online supplementary table 1. Significant heterogeneity in analytical framework and outcome measures used in these studies restricted meta-analysis and direct ranking of the interventions by their degree of cost-effectiveness.

\section{DISCUSSION}

This review finds that, with some exceptions, most interventions to control CVD and DM were cost-effective $(<1-3 \times$ GDP per capita per QALY gained or DALY averted), although the strength of evidence (and risk of bias) varied across economic evaluations based on observational studies, RCTs and decision models. Most interventions were cost-effective because of the large benefits in DALY averted or QALY gained at a marginal increase in cost per capita (\$). These results should motivate decision makers to invest in primordial prevention strategies (increased tobacco tax, salt reduction by legislation, food labelling and food advertising regulation), and primary and secondary prevention interventions: multidrug therapy for CVD prevention and treatment in high-risk groups, lifestyle modification and metformin for diabetes prevention, and screening for diabetes complications every $2-5$ years. Although detecting and treating diabetes earlier can prevent future complications and their associated medical costs, such savings were shown to be relatively small. ${ }^{34}$ An alternative to broad screening is to focus on targeted screening, that is, screening only persons with additional risk factors, such as hypertension and obesity. Such targeted screening was shown to be highly cost-effective or cost-saving when compared with no screening. ${ }^{33}$

Choice of comparator is an important decision when evaluating ICER of new interventions. In general, modelling studies that used the WHO-CHOICE method have reported average cost-effectiveness ratio against the null scenario (no intervention). In reality, however, this does not seem plausible because null scenario will not always reflect zero costs and zero effects. Also, these studies first identified the most cost-effective intervention among a group of strategies (eg, tobacco control, CVD prevention and treatment, or diabetes prevention and treatment) versus null scenario, then compared it with the next most cost-effective intervention. ${ }^{29}$ In many of such analysis, because the description of comparator was not clearly specified, the reported ICERs look ambiguous and changing the 'comparator' might produce a different ICER.

In our formal appraisal of the methodological quality of studies, we observed limitations in documentation of main study details, for example, chosen study perspective, sources of cost data and analytical time horizon. In addition, significant number of studies failed to provide details on units of resource use, costing year, currencies and other economic aspects. Since the discount rate used has an impact on cost-effectiveness estimates, the zero-discount rate applied in some studies is deeply concerning. In reality, however, every economic evaluation will contain some degree of uncertainty or imprecision. While one-way sensitivity analysis is helpful in understanding the impact of assumptions about one input parameter, multi-way sensitivity analysis offers a robust method to explore the uncertainty concerning more than one input parameters, but few studies reported results using this technique.

In terms of comparing results of this review with other contemporary reviews, we found cost-effectiveness evidence on a large number of preventive strategies, which is inconsistent with a previous review that examined the economic evaluation from Health Economic Evaluation Database $^{42}$ and concluded that only $10 \%$ of all evaluations assessed preventive care. The greater number of preventive strategies found in our review could be due to the development of the WHO-CHOICE programme ${ }^{26}$ and the release of the DCPP2 in April 2006. ${ }^{43}$ 
Although cost-effectiveness evidence is available for 301 interventions to control CVD or DM, most of this evidence is based on decision models, which used data (annual risk of disease progression and intervention benefits) from Western countries. Most decision model studies have derived treatment effects from either meta-analysis of RCTs if available for an intervention or single RCT if meta-analysis is not available. However, the limited representation of South Asian populations in those RCTs remains an important concern. Therefore, our review highlights an alarming paucity of local research data to conduct high-quality economic evaluations and reflect the concerns of others in the field that large research gaps do remain in the area of health economic analysis in South Asian countries. ${ }^{44}$ Also, data from countries other than India are sparse. This is likely a reflection of research capacity in these countries, which needs to be addressed as a priority. Although the countries in South Asia are frequently grouped together, various countries in this region have substantially different health systems, health literacy, health indices, and hence healthcare needs. Understanding the differences be the countries is critical for policy makers, and therefore additional economic evaluations are urgently needed from other South Asian countries.

\section{Strengths and limitations}

This review has several strengths. This is the first study, to our knowledge, to include all types of interventions (policy, clinical and behavioural) that affect CVD or DM in South Asia. We considered all possible interventions (primordial, primary, secondary and tertiary prevention) to control CVD and DM together in this systematic review, primarily because policy makers have to choose between different options (competing priorities) for appropriate resource allocation, and as such a narrow economic research question is really not helpful for the systematic review, which intends to inform the process. We have used explicitly stated methods (protocol paper published) ${ }^{19}$ and standard checklists to assess methodological quality of studies. Recently, new methods have been proposed by researchers that can be applied to review decision model studies. ${ }^{45}$ However, use of new criteria would not change the findings of this review because these points have been covered broadly by the three popular checklists that we used in this review. Also, new methods have been proposed to estimate country-specific threshold for cost-effectiveness based on opportunity cost (health forgone) with investment in new intervention. ${ }^{46}$ But we preferred to present the findings based on WHO guidelines ${ }^{25}$ and for a lower threshold, that is, $1 \times$ GDP per capita. Moreover, the incremental cost and incremental benefits have been shown for all interventions (where available) so the decision makers or clinicians can make considerations based on their own willingness to pay threshold or budgetary constraints.

This review is not without limitations. First, the search was restricted to English-language publications performed as of August 2016. But this would not be a major problem because all the South Asian countries mostly publish research in English. Second, we excluded unpublished and 'grey' literature as we wanted to include studies that have undergone peer review process. We believe though that no major studies that can change the results of this review have been missed.

The review findings should be interpreted with caution because most of the cost-effectiveness studies were based on decision models. Although good-quality decision modelling study can provide information at a lower cost than RCT-based economic evaluations, models are based on assumptions and represent a simplification of - and therefore might depart from-reality. Furthermore, interventions that were highlighted as cost-effective (yellow) or highly cost-effective or dominant (green) analysed using the WHO-CHOICE framework could be reassessed by local agencies, particularly with regard to budget impact and also their cost-effectiveness, taking into account local costs and willingness to pay threshold value, similar to the work carried out by the Health Intervention and Technology Assessment Program in Thailand over the past decade. ${ }^{47}$

\section{Future research directions}

We have identified key research gaps in this review. Interventions involving multisectoral approach and policies for change in drug prices or devices (stents prices) have not been evaluated for their cost-effectiveness. The cost-effectiveness of these interventions should be assessed.

A few recommendations to advance the research on economic evaluations in the region are as follows. First, future studies need to take a broader societal perspective for analysis and present cost data in disaggregated form (resource consumption and unit costs, separately). Second, more research is needed to support the causes of variation among costs, effects and cost-effectiveness data on the universal screening of diabetes and/or hypertension. Third, research should focus on assessing the generalisability of cost-effectiveness analysis results within and between countries. Lastly, future cost-effectiveness analysis studies should adhere to international guidelines proposed by the WHO, ${ }^{25}$ International Society for Pharmacoeconomics and Outcomes Research, ${ }^{48-51}$ and the recommendations of the Second Panel on Cost-Effectiveness in Health and Medicine ${ }^{52}$ as a benchmark for design, conduct and reporting.

\section{CONCLUSION}

The existing economic evidence base from South Asia should motivate policy makers to mobilise resource allocation towards the most cost-effective interventions identified in this review to curb the epidemic of CVD and DM in the region. Also, there is an urgent need to invest in health technology assessment and policy evaluations in South Asia using local research data. 
Author affiliations

${ }^{1}$ Department of Endocrinology and Metabolism, All India Institute of Medical Sciences, New Delhi, Delhi, India

${ }^{2}$ Clinical Trials Unit, Centre for Chronic Disease Control, New Delhi, Delhi, India

${ }^{3}$ Centre for Chronic Conditions and Injuries, Public Health Foundation of India, New Delhi, Delhi, India

${ }^{4}$ Health Promotion Division, Public Health Foundation of India, New Delhi, Delhi, India

${ }^{5}$ Division of Epidemiology and Public Health, School of Medicine, The University of Nottingham, Nottingham, UK

${ }^{6}$ Department of Non-communicable Disease Epidemiology, London School of Hygiene and Tropical Medicine, London, UK

${ }^{7}$ Management Development and Planning Unit, Ministry of Health, Colombo, Western, Sri Lanka

${ }^{8}$ WHO Collaborating Centre for Public Health Workforce Development, National Institute of Health Sciences, Kalutara, Sri Lanka

${ }^{9}$ Department of Cardiology, All India Institute of Medical Sciences, New Delhi, India

Contributors KS, NT, DP and AR conceptualised and designed the study. KS, AMCS and SB designed the search strategy for the review. KS and AMCS performed the search strategy in electronic databases, screened, reviewed and extracted data from eligible studies included in this review, and performed data analysis. KS wrote the first draft of the manuscript. AMCS, SB, KC, PDS, AUG, AR, DP and NT contributed significantly to the revision of the manuscript. All authors have approved the submission of this version of the manuscript.

Funding This research has received no specific grant from any funding agency in the public, commercial or not-for-profit sectors.

Competing interests None declared.

Patient consent Not required.

Provenance and peer review Not commissioned; externally peer reviewed.

Data sharing statement In this paper, we report the results of a systematic review. KS has access to all the data extracted from published studies. However, there are no unpublished data linked with this systematic review.

Open Access This is an Open Access article distributed in accordance with the Creative Commons Attribution Non Commercial (CC BY-NC 4.0) license, which permits others to distribute, remix, adapt, build upon this work non-commercially, and license their derivative works on different terms, provided the original work is properly cited and the use is non-commercial. See: http://creativecommons.org/ licenses/by-nc/4.0/

(C) Article author(s) (or their employer(s) unless otherwise stated in the text of the article) 2018. All rights reserved. No commercial use is permitted unless otherwise expressly granted.

\section{REFERENCES}

1. Ezzati M, Hoorn SV, Rodgers A, et al. Estimates of global and regional potential health gains from reducing multiple major risk factors. Lancet 2003;362:271-80.

2. Mendis S, Chestnov O. Costs, benefits, and effectiveness of interventions for the prevention, treatment, and control of cardiovascular diseases and diabetes in Africa. Prog Cardiovasc Dis 2013;56:314-21.

3. Tight blood pressure control and risk of macrovascular and microvascular complications in type 2 diabetes: UKPDS 38. UK Prospective Diabetes Study Group. BMJ 1998;317:703-13.

4. Yusuf S. Two decades of progress in preventing vascular disease. Lancet 2002;360:2-3.

5. Willett WC, Koplan JP, Nugent R. Disease control priorities in developing countries, 2nd edition. Chapter 44 prevention of chronic disease by means of diet and lifestyle changes. In: Jamison DT, Breman JG, Measham AR, Alleyne G, Claeson M, eds. Washington (DC): The International Bank for Reconstruction and Development / The World Bank. New York: Oxford University Press, 2006.

6. Wheeler ML. Translation of successful diabetes-related lifestyle interventions from research to practice. Curr Diab Rep 2005;5:363-5.

7. Webster R, Rodgers A. Polypill treatments for cardiovascular diseases. Expert Opin Drug Deliv 2016;13:1-6.

8. Turnbull F, Neal B, Ninomiya T, et al. Effects of different regimens to lower blood pressure on major cardiovascular events in older and younger adults: meta-analysis of randomised trials. BMJ 2008;336:1121-3.

9. Smith SC, Chen D, Collins A, et al. Moving from political declaration to action on reducing the global burden of cardiovascular diseases: a statement from the Global Cardiovascular Disease Taskforce. J Am Coll Cardiol 2013;62:2151-3.

10. Lee ES, Vedanthan R, Jeemon P, et al. Quality Improvement for Cardiovascular Disease Care in Low- and Middle-Income Countries: A Systematic Review. PLoS One 2016;11:e0157036.

11. Bhargava SK, Sachdev HS, Fall CH, et al. Relation of serial changes in childhood body-mass index to impaired glucose tolerance in young adulthood. N Engl J Med 2004;350:865-75.

12. Truelsen T, Bonita R, Grønbaek M, et al. Stroke incidence and case fatality in two populations: the Auckland Stroke Study and the Copenhagen City Heart Study. Neuroepidemiology 1998;17:132-8.

13. Ezzati M. Complexity and rigour in assessing the health dimensions of sectoral policies and programmes. Bull World Health Organ 2003;81:458-9.

14. Chisholm D, Sanderson K, Ayuso-Mateos JL, et al. Reducing the global burden of depression: population-level analysis of intervention cost-effectiveness in 14 world regions. Br J Psychiatry 2004;184:393-403.

15. Chisholm D, Rehm J, Van Ommeren M, et al. Reducing the global burden of hazardous alcohol use: a comparative cost-effectiveness analysis. J Stud Alcohol 2004;65:782-93.

16. Chisholm D, Doran C, Shibuya K, et al. Comparative costeffectiveness of policy instruments for reducing the global burden of alcohol, tobacco and illicit drug use. Drug Alcohol Rev 2006;25:553-65.

17. Shroufi A, Chowdhury R, Anchala R, et al. Cost effective interventions for the prevention of cardiovascular disease in low and middle income countries: a systematic review. BMC Public Health 2013;13:285.

18. Walker D, Fox-Rushby JA. Economic evaluation of communicable disease interventions in developing countries: a critical review of the published literature. Health Econ 2000;9:681-98.

19. Singh K, Chandra Sekaran AM, Bhaumik S, et al. Cost-effectiveness of interventions to control cardiovascular diseases and type 2 diabetes mellitus in South Asia: protocol for a systematic review. BMJ Open 2015;5:e007205.

20. Drummond MF, Sculpher MJ, Torrance GW, et al. Methodsfor the Economic Evaluation of Health Care Programs. 3rd edn. Oxford, UK: Oxford University Press, 2005.

21. Evers S, Goossens M, de Vet $\mathrm{H}$, et al. Criteria list for assessment of methodological quality of economic evaluations: consensus on health economic criteria. Int J Technol Assess Health Care 2005;21:240-5.

22. Philips Z, Ginnelly L, Sculpher M, et al. Review of guidelines for good practice in decision-analytic modelling in health technology assessment. Health Technol Assess 2004;8:iii-iv, ix-xi, 1-158.

23. Inflation Calculator India. Calculate India's inflation between any two years from 1971 to 2017. http://calculatorstack.com/inflationcalculator-india.php (accessed on 12 Aug 2017).

24. Inflation calculator. http://fxtop.com/en/inflation-calculator.php (accessed on 12 Aug 2017).

25. World Health Organization. WHO Guide to cost-effectiveness analysis. Geneva: World Health Organization, 2003.

26. Murray C, Lopez A, Mathers C, et al. The global burden of disease 2000 project: aims, methods and data sources. Geneva: World Health Organization, 2001.

27. Rachapelle S, Legood R, Alavi Y, et al. The cost-utility of telemedicine to screen for diabetic retinopathy in India. Ophthalmology 2013;120:566-73.

28. The World Bank Data. All countries and economies - GDP per capita http://data.worldbank.org/indicator/NY.GDP.PCAP.CD (accessed on 20 Aug 2017).

29. Ortegón M, Lim S, Chisholm D, et al. Cost effectiveness of strategies to combat cardiovascular disease, diabetes, and tobacco use in subSaharan Africa and South East Asia: mathematical modelling study. BMJ 2012;344:e607.

30. Donaldson EA, Waters HR, Arora M, et al. A cost-effectiveness analysis of India's 2008 prohibition of smoking in public places in Gujarat. Int J Environ Res Public Health 2011;8:1271-86.

31. Brown HS, Stigler M, Perry C, et al. The cost-effectiveness of a school-based smoking prevention program in India. Health Promot Int 2013;28:178-86.

32. Cecchini M, Sassi F, Lauer JA, et al. Tackling of unhealthy diets, physical inactivity, and obesity: health effects and cost-effectiveness. Lancet 2010;376:1775-84.

33. Dukpa W, Teerawattananon Y, Rattanavipapong W, et al. Is diabetes and hypertension screening worthwhile in resource-limited settings? 
An economic evaluation based on a pilot of a package of essential non-communicable disease interventions in Bhutan. Health Policy Plan 2015;30:1032-43.

34. Narayan KM, Zhang P, Kanaya AM. Disease Control Priorities in Developing Countries, 2nd edition. Chapter 30 Diabetes: The Pandemic and Potential Solutions. In: Jamison DT, Breman JG, Measham AR, Alleyne G, Claeson M, eds. Washington (DC): the international bank for reconstruction and development / The World Bank. New York: Oxford University Press, 2006.

35. Marseille E, Lohse N, Jiwani A, et al. The cost-effectiveness of gestational diabetes screening including prevention of type 2 diabetes: application of a new model in India and Israel. J Matern Fetal Neonatal Med 2013;26:802-10.

36. Jafar TH, Islam M, Bux R, et al. Cost-effectiveness of communitybased strategies for blood pressure control in a low-income developing country: findings from a cluster-randomized, factorialcontrolled trial. Circulation 2011;124:1615-25.

37. Ramachandran A, Snehalatha C, Yamuna A, et al. Cost-effectiveness of the interventions in the primary prevention of diabetes among Asian Indians: within-trial results of the Indian Diabetes Prevention Programme (IDPP). Diabetes Care 2007;30:2548-52.

38. Megiddo I, Chatterjee S, Nandi A, et al. Cost-effectiveness of treatment and secondary prevention of acute myocardial infarction in India: a modeling study. Glob Heart 2014;9:391-8.

39. Basu S, Bendavid E, Sood N. Health and economic implications of national treatment coverage for cardiovascular disease in india: cost-effectiveness analysis. Circ Cardiovasc Qual Outcomes 2015;8:541-51.

40. Schulman-Marcus J, Prabhakaran D, Gaziano TA. Pre-hospital ECG for acute coronary syndrome in urban India: a cost-effectiveness analysis. BMC Cardiovasc Disord 2010;10:13.

41. Gupta V, Baabbad R, Hammerby E, et al. An analysis of the costeffectiveness of switching from biphasic human insulin 30 , insulin glargine, or neutral protamine Hagedorn to biphasic insulin aspart 30 in people with type 2 diabetes. J Med Econ 2015;18:263-72.

42. Pritchard C. Office of Health Economics Briefing. Trends in Economic Evaluation. 1998.

43. Gaziano TA, Reddy KS, Paccaud F. Disease Control Priorities in Developing Countries, 2nd edition. Chapter 33 Cardiovascular Disease. In: Jamison DT, Breman JG, Measham AR, Alleyne G, Claeson $\mathrm{M}$, eds. Washington (DC): the international bank for reconstruction and development / The World Bank. New York: Oxford University Press, 2006.

44. Mendis S, Fukino K, Cameron A, et al. The availability and affordability of selected essential medicines for chronic diseases in six low- and middle-income countries. Bull World Health Organ 2007;85:279-88.

45. Epstein D, García-Mochón L, Kaptoge S, et al. Modeling the costs and long-term health benefits of screening the general population for risks of cardiovascular disease: a review of methods used in the literature. Eur J Health Econ 2016;17:1041-53.

46. Woods B, Revill P, Sculpher M, et al. Country-level cost-effectiveness thresholds: initial estimates and the need for further research. Value Health 2016;19:929-35.

47. Health intervention and technology assessment program, Thailand. $2007 \mathrm{http}: / /$ www.hitap.net/en/ (accessed on 05 Dec 2017).

48. Ramsey SD, Willke RJ, Glick H, et al. Cost-effectiveness analysis alongside clinical trials II-An ISPOR Good Research Practices Task Force report. Value Health 2015;18:161-72.

49. Ramsey S, Willke R, Briggs A, et al. Good research practices for cost-effectiveness analysis alongside clinical trials: the ISPOR RCTCEA Task Force report. Value Health 2005;8:521-33.

50. Weinstein MC, O'Brien B, Hornberger J, et al. Principles of good practice for decision analytic modeling in health-care evaluation: report of the ISPOR Task Force on Good Research Practices-Modeling Studies. Value Health 2003;6:9-17.

51. Garrison LP. The ISPOR good practice modeling principles-a sensible approach: be transparent, be reasonable. Value Health 2003;6:6-8.

52. Bibbins-Domingo K, Grossman DC, Curry SJ, et al. Statin use for the primary prevention of cardiovascular disease in adults: US preventive services task force recommendation statement. JAMA 2016;316:1997-2007.

53. Turi ZG, Reyes VP, Raju BS, et al. Percutaneous balloon versus surgical closed commissurotomy for mitral stenosis. A prospective, randomized trial. Circulation 1991;83:1179-85.

54. Ahuja RC, Mitra MK, Saran RK, et al. A RCT to estimate cost effectiveness of antihypertensive regimes with or without diuretics for management of mild hypertension. J Clin Epidemiol 1997;50:S25.

55. Nanjappa MC, Dorros G, Hemanna Setty SK, et al. The Indian Experience of Percutaneous Transvenous Mitral Commissurotomy:
Comparison of the Triple Lumen (Inoue) and Double Lumen (Accura) Variable Sized Single Balloon With Regard to Procedural Outcome and Cost Savings. J Interv Cardiol 1998;11:107-12.

56. Malhotra S, Bhargava VK, Grover A, et al. A randomized trial to compare the efficacy, safety, cost and platelet aggregation effects of enoxaparin and unfractionated heparin (the ESCAPEU trial). Int J Clin Pharmacol Ther 2001;39:110-5.

57. Murray CJ, Lauer JA, Hutubessy RC, et al. Effectiveness and costs of interventions to lower systolic blood pressure and cholesterol: a global and regional analysis on reduction of cardiovascular-disease risk. Lancet 2003;361:717-25.

58. Namboodiri KK, Sharma YP, Bali HK, et al. Re-use of explanted DDD pacemakers as VDD- clinical utility and cost effectiveness. Indian Pacing Electrophysiol J 2004;4:3-9.

59. Rodgers A, Lawes CMM, Gaziano TA. Disease Control Priorities in Developing Countries, 2nd edition. Chapter 45 The Growing Burden of Risk from High Blood Pressure, Cholesterol, and Bodyweight. In: Jamison DT, Breman JG, Measham AR, Alleyne G, Claeson M, eds. Washington (DC): the international bank for reconstruction and development / The World Bank. New York: Oxford University Press, 2006.

60. Jha P, Chaloupka FJ, Moore J. et a/Disease Control Priorities in Developing Countries, 2nd edition. Chapter 46 Tobacco Addiction. In: Jamison DT, Breman JG, Measham AR, Alleyne G, Claeson M, eds. Washington (DC): the international bank for reconstruction and development / The World Bank. New York: Oxford University Press, 2006.

61. Shafiq N, Malhotra S, Pandhi P, et al. A randomized controlled clinica trial to evaluate the efficacy, safety, cost-effectiveness and effect on PAl-1 levels of the three low-molecular-weight heparins-enoxaparin, nadroparin and dalteparin. The ESCAPe-END study. Pharmacology 2006;78:136-43.

62. Zubair Tahir M, Enam SA, Pervez Ali R, et al. Cost-effectiveness of clipping vs coiling of intracranial aneurysms after subarachnoid hemorrhage in a developing country-a prospective study. Surg Neurol 2009;72:355-60.

63. Habib SH, Akter S, Saha S, et al. Cost-effectiveness analysis of medical intervention in patients with early detected of Diabetic Nephropathy in a tertiary care hospital in Bangladesh. Diabetes \& Metabolic Syndrome. Clinical Research \& Reviews 2010.

64. Habib SH, Biswas KB, Akter S, et al. Cost-effectiveness analysis of medical intervention in patients with early detection of diabetic foot in a tertiary care hospital in Bangladesh. $J$ Diabetes Complications 2010;24:259-64.

65. Sanmukhani J, Shah V. Statins: cost analysis in Indian scenario from eight major clinical trials. J Postgrad Med 2010;56:196-200.

66. Lohse N, Marseille E, Kahn JG. Development of a model to assess the cost-effectiveness of gestational diabetes mellitus screening and lifestyle change for the prevention of type 2 diabetes mellitus. Int $J$ Gynaecol Obstet 2011;115(Suppl 1):S20-5.

67. Ahmad M, Wafai ZA, Khan ZY, et al. Evaluation of the CostEffectiveness of Different Insulin Regimes during the PeriOperative Period in Type-2 Diabetics in India. J Clin Diagn Res 2011;5:1064-8.

68. Humaira S. Cost-effectiveness of medical intervention in patients with early detection of diabetic retinopathy in a tertiary care hospital in Bangladesh. The Experiment 2012;5:293-303.

69. Patel RS, Sharma KH, Kamath NA, et al. Cost-effectiveness analysis of nebivolol and metoprolol in essential hypertension: a pharmacoeconomic comparison of antihypertensive efficacy of beta blockers. Indian J Pharmacol 2014;46:485-9.

70. Lamy A, Tong W, Jung $\mathrm{H}$, et al. Cost implications of the use of basal insulin glargine in people with early dysglycemia: the ORIGIN trial. $J$ Diabetes Complications 2014;28:553-8.

71. Lamy A, Tong W, Devereaux PJ, et al. The cost implications of offpump versus on-pump coronary artery bypass graft surgery at one year. Ann Thorac Surg 2014;98:1620-5.

72. Anchala R, Kaptoge S, Pant $\mathrm{H}$, et al. Evaluation of effectiveness and cost-effectiveness of a clinical decision support system in managing hypertension in resource constrained primary health care settings: results from a cluster randomized trial. J Am Heart Assoc 2015;4:e001213.

73. Dukpa W, Teerawattananon $\mathrm{Y}$, Rattanavipapong W, et al. Is diabetes and hypertension screening worthwhile in resource-limited settings? An economic evaluation based on a pilot of a Package of Essential Non-communicable disease interventions in Bhutan. Health Policy Plan 2015;30:1032-43.

74. Basu S, Millett C, Vijan S, et al. The health system and population health implications of large-scale diabetes screening in India: a microsimulation model of alternative approaches. PLoS Med 2015;12:e1001827. 
75. Home P, Baik SH, Gálvez GG, et al. An analysis of the costeffectiveness of starting insulin detemir in insulin-naïve people with type 2 diabetes. J Med Econ 2015;18:230-40.

76. Sengottuvelu G, Chakravarthy B, Rajendran R, et al. Clinical usefulness and cost effectiveness of fractional flow reserve among Indian patients (FIND study). Catheter Cardiovasc Interv 2016;88:E139-E144.

77. Limaye D, Todi K, Shroff J, et al. Cost-effectiveness study of antidiabetic drugs in type 2 diabetes mellitus patients from Mumbai, India. Current Therapeutic Research 2016;78:S2-S3.
78. Basu S, Yudkin JS, Sussman JB, et al. Alternative strategies to achieve cardiovascular mortality goals in China and India: a microsimulation of target- versus risk-based blood pressure treatment. Circulation 2016;133:840-8.

79. Mathers CD, Loncar D. Projections of global mortality and burden of disease from 2002 to 2030. PLoS Med 2006;3:e442

80. McFayden JE. ed. International drug price indicator reference guide: Boston Management Sciences for Health, 2003. 